This is an Open Access article, distributed under the terms of the Creative Commons Attribution licence (http://creativecommons.org/licenses/by/4.0/), which permits unrestricted re-use, distribution, and reproduction in any medium, provided the original work is properly cited.

doi:10.1017/jfm.2019.476

\title{
Constitutive relations for compressible granular flow in the inertial regime
}

\author{
D. G. Schaeffer ${ }^{1}$, T. Barker ${ }^{2}$, D. Tsuji ${ }^{3}$, P. Gremaud ${ }^{4}$, M. Shearer ${ }^{4}$ \\ and J. M. N. T. Gray ${ }^{2, \dagger}$ \\ ${ }^{1}$ Mathematics Department, Duke University, Box 90320, Durham, NC 27708-0320, USA \\ ${ }^{2}$ School of Mathematics and Manchester Centre for Nonlinear Dynamics, University of Manchester, \\ Oxford Road, Manchester M13 9PL, UK \\ ${ }^{3}$ Department of Earth and Environmental Sciences, Nagoya University, Furocho, Chikusa, \\ Nagoya 464-8601, Japan \\ ${ }^{4}$ Department of Mathematics, North Carolina State University, Raleigh, NC 27695-8205, USA
}

(Received 18 December 2018; revised 8 June 2019; accepted 8 June 2019; first published online 15 July 2019)

Granular flows occur in a wide range of situations of practical interest to industry, in our natural environment and in our everyday lives. This paper focuses on granular flow in the so-called inertial regime, when the rheology is independent of the very large particle stiffness. Such flows have been modelled with the $\mu(I), \Phi(I)$-rheology, which postulates that the bulk friction coefficient $\mu$ (i.e. the ratio of the shear stress to the pressure) and the solids volume fraction $\phi$ are functions of the inertial number $I$ only. Although the $\mu(I), \Phi(I)$-rheology has been validated in steady state against both experiments and discrete particle simulations in several different geometries, it has recently been shown that this theory is mathematically ill-posed in time-dependent problems. As a direct result, computations using this rheology may blow up exponentially, with a growth rate that tends to infinity as the discretization length tends to zero, as explicitly demonstrated in this paper for the first time. Such catastrophic instability due to ill-posedness is a common issue when developing new mathematical models and implies that either some important physics is missing or the model has not been properly formulated. In this paper an alternative to the $\mu(I), \Phi(I)$-rheology that does not suffer from such defects is proposed. In the framework of compressible $I$-dependent rheology (CIDR), new constitutive laws for the inertial regime are introduced; these match the well-established $\mu(I)$ and $\Phi(I)$ relations in the steady-state limit and at the same time are well-posed for all deformations and all packing densities. Time-dependent numerical solutions of the resultant equations are performed to demonstrate that the new inertial CIDR model leads to numerical convergence towards physically realistic solutions that are supported by discrete element method simulations.

Key words: granular media, rheology

$\dagger$ Email address for correspondence: nico.gray@manchester.ac.uk 


\section{Introduction}

The original incompressible $\mu(I)$-rheology was proposed (GDR MiDi 2004; Jop, Forterre \& Pouliquen 2006) to describe granular flow in which the particles are rigid and the solids volume fraction $\phi$ is constant and uniform. The key physical insight behind the theory was that, under these circumstances, the only non-dimensional groups are the bulk friction coefficient $\mu$ (the ratio $\tau / p$ of shear stress to pressure) and the inertial number

$$
I=\frac{d \dot{\gamma}}{\sqrt{p / \rho_{*}}},
$$

where $d$ is the grain diameter, $\dot{\gamma}$ is the shear rate, $p$ is the pressure and $\rho_{*}$ is the intrinsic grain density. When forming a constitutive law from these groups, dimensionless analysis implies that one group must be a function of the other, i.e.

$$
\frac{\tau}{p}=\mu(I)
$$

This approach has led to significant progress in modelling granular flows (e.g. Lagrée, Staron \& Popinet 2011; Staron, Lagrée \& Popinet 2012; Gray \& Edwards 2014; Barker \& Gray 2017). However, Barker et al. (2015) identified conditions on $\mu(I)$ and $I$ under which incompressible $\mu(I)$-rheology is ill-posed.

In reality granular flow is compressible and the solids volume fraction $\phi$ may vary. With compressibility there are multiple non-dimensional groups, which greatly complicates the possible rheology. Nevertheless, GDR MiDi (2004), da Cruz et al. (2005) and Pouliquen et al. (2006) found that, in steady-state simulations and experiments, $\phi$ depended on just the inertial number

$$
\phi=\Phi(I)
$$

where $\Phi$ is a monotonically decreasing function of $I$. Note that (1.3) implies Bagnold scaling (Bagnold 1954) for the pressure (i.e. it scales with the square of strain rate). Specifically, if $\Psi(\phi)$ is the inverse function of $\Phi(I)$, then (1.1) may be rearranged to yield

$$
p=\rho_{*}\left(\frac{d \dot{\gamma}}{\Psi(\phi)}\right)^{2} .
$$

This is consistent with the discrete element method (DEM) simulations of Chialvo, Sun \& Sundaresan (2012) in the inertial regime, in which the solids volume fraction $\phi$ is less than a critical volume fraction $\phi_{c}$, namely the jamming point (Liu \& Nagel 1998). For $\phi>\phi_{c}$, the stiffness of the particles becomes important and the rheology changes to either a 'quasi-static' or an 'intermediate' regime, which both depart from the Bagnold scaling. In general, the critical volume fraction $\phi_{c}$ is dependent on the polydispersity of the grain-size distribution as well as the interparticle friction.

These observations led to the compressible $\mu(I), \Phi(I)$-rheology (GDR MiDi 2004; Pouliquen et al. 2006; Forterre \& Pouliquen 2008) in which the scalar relation (1.3), as well as (1.2), is assumed to hold even in non-steady situations. Although it might seem that compressibility would reduce the tendency to ill-posedness, in fact the compressible $\mu(I), \Phi(I)$-rheology is even more prone to ill-posedness in time-dependent calculations than incompressible $\mu(I)$-rheology (Heyman et al. 2017). Such instability is perhaps not surprising since the equations are not always dissipative (see appendix B). To demonstrate this ill-posedness, $\S 3$ presents computations for 
a one-dimensional gravity-free shear cell in which the $\mu(I), \Phi(I)$-rheology blows up catastrophically once the mesh size is refined sufficiently, even though the initial conditions are just a small perturbation of the steady solution.

The purpose of this paper is to develop a viable alternative to $\mu(I), \Phi(I)$-rheology that preserves its successes. This alternative is based on the compressible $I$-dependent rheology (CIDR) introduced recently in Barker et al. (2017). After briefly recalling the general formulation of CIDR, specific yield and dilatancy functions are introduced, which ensure that the theory is well-posed and reduces to $\mu(I), \Phi(I)$-rheology in the correct limits. Specifically, the new inertial CIDR equations recover both the $\mu(I)$ and $\Phi(I)$ relations (1.2)-(1.3) when the flow is isochoric (div $\boldsymbol{u}=0)$, which in many geometries corresponds to steady flow. Sample computations with the one-dimensional inertial CIDR equations in a gravity-free shear cell converge to the steady-state solution, even for initial conditions that are a long way from steady state. Moreover, these computations agree well with transient DEM simulations of the same flows.

In $\S \S 2$ and 3 the $\mu(I), \Phi(I)$-rheology is described and computations that blow up because of ill-posedness are presented. Sections 4 and 5 introduce CIDR for the inertial regime and show that computations converge to steady state, and these computations are supported by DEM simulations described in $\S 6$. The final two sections discuss a number of related issues and summarize the overall conclusions. Appendix A derives conditions for ill-posedness of the one-dimensional $\mu(I), \Phi(I)$-rheology in a gravity-free shear cell, appendix B relates the CIDR equations to the thermodynamic analysis of Goddard \& Lee (2018) and appendix C describes the formulation of the DEM simulations.

\section{The $\mu(I), \Phi(I)$-rheology for granular flows}

In a continuum formulation, dense granular flow is described by the solids fraction $\phi$, the velocity vector $\boldsymbol{u}$ and the symmetric Cauchy stress tensor $\boldsymbol{\sigma}$. In two dimensions (to which this paper is restricted) this formulation constitutes six scalar unknown functions of position and time. These satisfy governing equations including three conservation laws: one for conservation of mass

$$
\left(\partial_{t}+u_{j} \partial_{j}\right) \phi+\phi \operatorname{div} \boldsymbol{u}=0,
$$

and two momentum conservation laws

$$
\rho_{*} \phi\left(\partial_{t}+u_{j} \partial_{j}\right) u_{i}=\partial_{j} \sigma_{i j}, \quad i=1,2,
$$

where $\rho_{*}$ is the intrinsic density of the grains and body forces have been neglected. In addition to the three conservation laws, three constitutive relations are needed to close this system.

To write these constitutive laws, it is convenient to decompose the stress tensor into a pressure term $p=-\sigma_{i i} / 2$ plus a trace-free tensor $\tau$, called the shear-stress tensor or the deviatoric stress tensor, such that

$$
\sigma_{i j}=-p \delta_{i j}+\tau_{i j} .
$$

The constitutive law for the incompressible $\mu(I)$-rheology (Jop et al. 2006; Forterre \& Pouliquen 2008; Barker et al. 2015) is formulated in terms of the (total) strain-rate tensor

$$
D_{i j}=\frac{1}{2}\left(\partial_{j} u_{i}+\partial_{i} u_{j}\right),
$$


but for the compressible generalizations of the $\mu(I)$-rheology (GDR MiDi 2004; Pouliquen et al. 2006; Forterre \& Pouliquen 2008; Barker et al. 2017; Heyman et al. 2017; Goddard \& Lee 2018) it is also useful to define the deviatoric strain-rate tensor

$$
S_{i j}=D_{i j}-\frac{1}{2}(\operatorname{div} \boldsymbol{u}) \delta_{i j},
$$

where $S_{i j}$ is trace free. The alignment constitutive law, which is imposed in both $\mu(I), \Phi(I)$-rheology and CIDR, states that

$$
\frac{S_{i j}}{\|\boldsymbol{S}\|}=\frac{\tau_{i j}}{\|\boldsymbol{\tau}\|}
$$

where the notation $\|\boldsymbol{T}\|=\sqrt{\operatorname{tr}\left(\boldsymbol{T}^{2}\right) / 2}$ denotes the second invariant of any symmetric tensor $\boldsymbol{T}$. In particular, the eigenvectors of the deviatoric stress tensor and the deviatoric strain-rate tensor are parallel. This matrix equation is in fact equivalent to just one scalar equation and relies upon the implicit assumption that $\|\boldsymbol{S}\| \neq 0$; i.e. that material is actually shearing. The other two constitutive laws in the $\mu(I), \Phi(I)$-rheology specify the deviatoric stress $\tau=\|\boldsymbol{\tau}\|$ and the solids fraction $\phi$ :

$$
\begin{gathered}
\tau=\mu(I) p, \\
\phi=\Phi(I),
\end{gathered}
$$

in terms of the pressure $p$ and the inertial number

$$
I=\frac{2 d\|\boldsymbol{S}\|}{\sqrt{p / \rho_{*}}}
$$

where $\mu(I)$ is the bulk friction coefficient and $d$ is the grain diameter.

The constitutive laws (2.6)-(2.8) are referred to as $\mu(I), \Phi(I)$-rheology. Commonly used functional forms for $\mu(I)$ and $\Phi(I)$ in the constitutive laws (2.7) and (2.8) are

$$
\begin{gathered}
\mu(I)=\mu_{s}+\frac{\mu_{d}-\mu_{s}}{I_{0} / I+1}, \\
\Phi(I)=\phi_{c}-a I,
\end{gathered}
$$

where $\mu_{s}, \mu_{d}, I_{0}, \phi_{c}$ and $a$ are constant material parameters (GDR MiDi 2004; Jop et al. 2006; Forterre \& Pouliquen 2008; Trulsson et al. 2013). As shown in figure 1, these functions provide a good fit for the DEM simulations performed in this paper (see appendix C). Table 1 lists the best-fit parameters extracted from the steady-state DEM simulations. Note that figure 1 includes data for multiple cases with differing particle stiffness and system size, which shows that the parameters do not depend on the details of the DEM simulations.

Incidentally the form $\Phi(I)=\phi_{c}-\left(\phi_{c}-\phi_{\min }\right) /\left(I_{*} / I+1\right)$, where $\phi_{\min }$ and $I_{*}$ are constants, might be preferable to (2.11), which becomes negative for large $I$. However, the simpler form (2.11) is adequate for most practical purposes, because $I$ rarely becomes large enough to drive $\Phi(I)$ negative.

Since (2.11) is a monotonically decreasing function, equation (2.8) can be inverted to write the inertial number as a function of the solids volume fraction

$$
I=\Psi(\phi),
$$



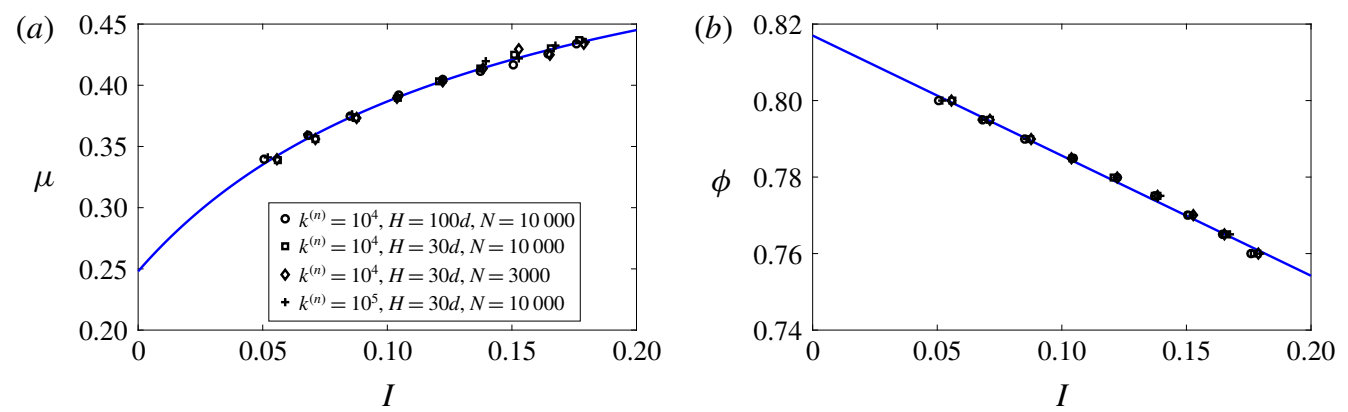

FIGURE 1. Steady-state relations for $(a)$ the bulk friction coefficient $\mu$ and $(b)$ the solids volume fraction $\phi$ as functions of the inertial number $I$. Solid blue lines are the functional forms (2.10) and (2.11) evaluated with the parameters in table 1. Symbols are the results of multiple DEM simulations (see appendix $\mathrm{C}$ for details) in which the normal spring constant $k^{(n)}$, the cell height $H$ and number of particles $N$ have been varied.

$$
\mu_{s}=0.248 \quad \mu_{d}=0.588 \quad I_{0}=0.145 \quad \phi_{c}=0.817 \quad a=0.314
$$

TABLE 1. The parameters in the constitutive laws (2.10) and (2.11) which are the best-fit parameters for the DEM data plotted in figure 1.

which, for the specific function (2.11) used here, gives

$$
\Psi(\phi)=\frac{\phi_{c}-\phi}{a}
$$

From this it is also possible to determine an equation of state for the pressure by substituting (2.12) into the inertial number (2.9) and solving for $p$ to give

$$
p=\rho_{*}\left(\frac{2 d\|\boldsymbol{S}\|}{\Psi(\phi)}\right)^{2},
$$

which, for fixed $\phi$, exhibits the Bagnold scaling in that the pressure scales with the square of strain rate as in (1.4). Note that this pressure tends to infinity as $\phi \rightarrow \phi_{c}$, behaviour that is discussed in $\S 7.2$.

\section{Catastrophic failure with the $\mu(I), \Phi(I)$-rheology}

As mentioned in the introduction, it follows from Heyman et al. (2017) that the dynamic equations of $\mu(I), \Phi(I)$-rheology for two-dimensional flow are always illposed. In this section it is demonstrated that ill-posedness can contaminate even the simplest of problems: flow in a two-dimensional shear cell that depends on only one spatial variable.

\subsection{Equations for one-dimensional flow in a shear cell}

In a planar shear cell granular material is confined from above and below by two parallel flat frictional walls, whose relative motion, in the absence of gravity, provides 
the only driving for the flow. This is a popular idealized geometry for the study of granular rheology, and the set-up can be either volume controlled, by fixing the wall separation distance $H$, or pressure controlled, by applying a normal force at the walls. In the following investigations $H$ will be fixed and solutions will be restricted to those which are invariant in the shearing direction $x$ and depend only on the vertical coordinate $z$ and time. As such, this reduces the two-dimensional problem to one spatial dimension in the interval $0<z<H$. As the flow is taken to be compressible, both components of the velocity $\boldsymbol{u}=(u, w)$ may be non-zero and depend on $z$ and $t$. For this special class of flows, the conservation laws (2.1)-(2.2) simplify to

$$
\begin{gathered}
\left(\partial_{t}+w \partial_{z}\right) \phi=-\phi \partial_{z} w, \\
\rho_{*} \phi\left(\partial_{t}+w \partial_{z}\right) u=\partial_{z} \tau_{x z}, \\
\rho_{*} \phi\left(\partial_{t}+w \partial_{z}\right) w=-\partial_{z} p+\partial_{z} \tau_{z z},
\end{gathered}
$$

since all $x$-derivatives vanish. In addition, the deviatoric strain rate reduces to

$$
\boldsymbol{S}=\frac{1}{2}\left[\begin{array}{cc}
-\partial_{z} w & \partial_{z} u \\
\partial_{z} u & \partial_{z} w
\end{array}\right],
$$

and its second invariant becomes

$$
\|\boldsymbol{S}\|=\frac{1}{2} \sqrt{\left(\partial_{z} u\right)^{2}+\left(\partial_{z} w\right)^{2}} .
$$

It follows that in the shear cell the equation of state (2.14) reduces to

$$
p=\frac{\rho_{*} d^{2}}{\Psi^{2}(\phi)}\left[\left(\partial_{z} u\right)^{2}+\left(\partial_{z} w\right)^{2}\right],
$$

which provides an explicit expression for the pressure.

The alignment condition (2.6) implies that the relevant components of the deviatoric stress are

$$
\left[\begin{array}{c}
\tau_{x z} \\
\tau_{z z}
\end{array}\right]=\frac{\|\boldsymbol{\tau}\|}{\|\boldsymbol{S}\|}\left[\begin{array}{l}
S_{x z} \\
S_{z z}
\end{array}\right],
$$

which using the constitutive law (2.7) and equations (3.5) and (2.12) implies

$$
\left[\begin{array}{c}
\tau_{x z} \\
\tau_{z z}
\end{array}\right]=\frac{\mu(\Psi(\phi)) p}{\sqrt{\left(\partial_{z} u\right)^{2}+\left(\partial_{z} w\right)^{2}}}\left[\begin{array}{c}
\partial_{z} u \\
\partial_{z} w
\end{array}\right] .
$$

Equations (3.6) and (3.8) may then be substituted into the conservation laws (3.1)(3.3) to obtain a system of three partial differential equations (PDEs) for $\phi, u$ and $w$ that are first order in time and second order in space.

For a given set of initial conditions $\phi(z, 0), u(z, 0), w(z, 0)$ the three PDEs must be solved subject to the boundary conditions that there is no slip at the walls

$$
u(H, t)=V_{0}, \quad w(H, t)=0, \quad u(0, t)=0, \quad w(0, t)=0,
$$

where $V_{0}$ is the velocity of the top wall. It is easily verified that the steady linear shearing solution

$$
\phi(z)=\phi_{0}, \quad u(z)=V_{0} \frac{z}{H}, \quad w(z)=0,
$$

where $\phi_{0}$ is the average initial solids volume fraction

$$
\phi_{0}=\frac{1}{H} \int_{0}^{H} \phi(z, 0) \mathrm{d} z
$$

satisfies (3.1)-(3.3) given (3.6), (3.8) and the boundary conditions (3.9). It is therefore anticipated that any set of initial conditions will tend to this solution. 
3.2. Blow up and grid dependence with the $\mu(I), \Phi(I)$-rheology

For one-dimensional flow in a shear cell, the physical variables are non-dimensionalized with the scalings

$$
z=d \hat{z}, \quad t=\left(d / V_{0}\right) \hat{t}, \quad p=\rho_{*} V_{0}^{2} \hat{p}, \quad(u, w)=V_{0}(\hat{u}, \hat{w})
$$

where the hats indicate non-dimensional variables. Equations (3.1)-(3.3) with the relations (3.6) and (3.8) therefore reduce to the non-dimensional system

$$
\begin{gathered}
\left(\partial_{\hat{t}}+\hat{w} \partial_{\hat{z}}\right) \phi=-\phi \partial_{\hat{z}} \hat{w}, \\
\phi\left(\partial_{\hat{t}}+\hat{w} \partial_{\hat{z}}\right) \hat{u}=\partial_{\hat{z}}\left(\frac{\mu(\Psi(\phi))}{\Psi^{2}(\phi)} \sqrt{\left(\partial_{\hat{z}} \hat{u}\right)^{2}+\left(\partial_{\hat{z}} \hat{w}\right)^{2}} \partial_{\hat{z}} \hat{u}\right), \\
\phi\left(\partial_{\hat{t}}+\hat{w} \partial_{\hat{z}}\right) \hat{w}=-\partial_{\hat{z}}\left(\frac{\left(\partial_{\hat{z}} \hat{u}\right)^{2}+\left(\partial_{\hat{z}} \hat{w}\right)^{2}}{\Psi^{2}(\phi)}\right)+\partial_{\hat{z}}\left(\frac{\mu(\Psi(\phi))}{\Psi^{2}(\phi)} \sqrt{\left(\partial_{\hat{z}} \hat{u}\right)^{2}+\left(\partial_{\hat{z}} \hat{w}\right)^{2}} \partial_{\hat{z}} \hat{w}\right),
\end{gathered}
$$

where the grains size $d$, the grain density $\rho_{*}$ and the wall velocity $V_{0}$ scale completely out of the system.

The conservation laws (3.13)-(3.15) are solved by the numerical method of lines (Schiesser 2012). This method discretizes the spatial derivatives in the PDEs which generates a system of coupled ordinary differential equations. These are then integrated forward in time using MATLAB's ODE15s routine. Two different methods are used to approximate the spatial derivatives: the first is a finite difference method using first-order differences, while the second method uses a Chebyshev spectral scheme (Canuto et al. 1988; Trefethen 2000).

The non-dimensional height of the cell $\hat{H}$ is chosen to equal 30 (i.e. the physical height of the cell is 30 grain diameters). The initial conditions are plotted in figure 2 and are identical to the non-dimensionalized steady solution except that the vertical velocity has a small smooth imperfection in the centre of the domain:

$$
\phi(\hat{z})=\phi_{0}, \quad \hat{u}(\hat{z})=\frac{\hat{z}}{\hat{H}}, \quad \hat{w}(\hat{z}, 0)=\epsilon\left(\hat{z}-\frac{\hat{H}}{2}\right) \mathrm{e}^{-(\hat{z}-\hat{H} / 2)^{2}}
$$

where $\epsilon$ is a small parameter. It should be noted that this form does not satisfy the boundary conditions exactly but does within numerical precision. Provided $\epsilon$ is not too small, the ill-posedness condition (A 19), outlined in appendix A, is satisfied in the centre of the domain, as shown in figure $2(d)$, and it is therefore anticipated that numerical problems will develop.

Confirming this anticipation, various snapshots of the non-dimensional vertical velocity are shown in figure 3 . (The full time evolutions of $\hat{u}(\hat{z}, \hat{t}), \hat{w}(\hat{z}, \hat{t})$ and $\phi(\hat{z}, \hat{t})$ are shown in supplementary movies 1-3.) In the high-resolution plots (figure $3 a, b$ ), the growth of noise causes the numerical method to break down at the indicated time - at this point integration tolerances can no longer be met. Note that the misbehaviour originates in the centre of the domain, where the ill-posedness condition (A 19) is satisfied. Moreover, although the two solutions blow up at similar times, their spatial structure is completely different.

In contrast, a low-resolution $\left(N_{z}=47\right)$ simulation using the finite difference scheme does not blow up and in fact decays towards the steady state, as shown in figures 3(c) and $4(a)$. (The same occurs for the low-resolution run with the Chebyshev spectral 

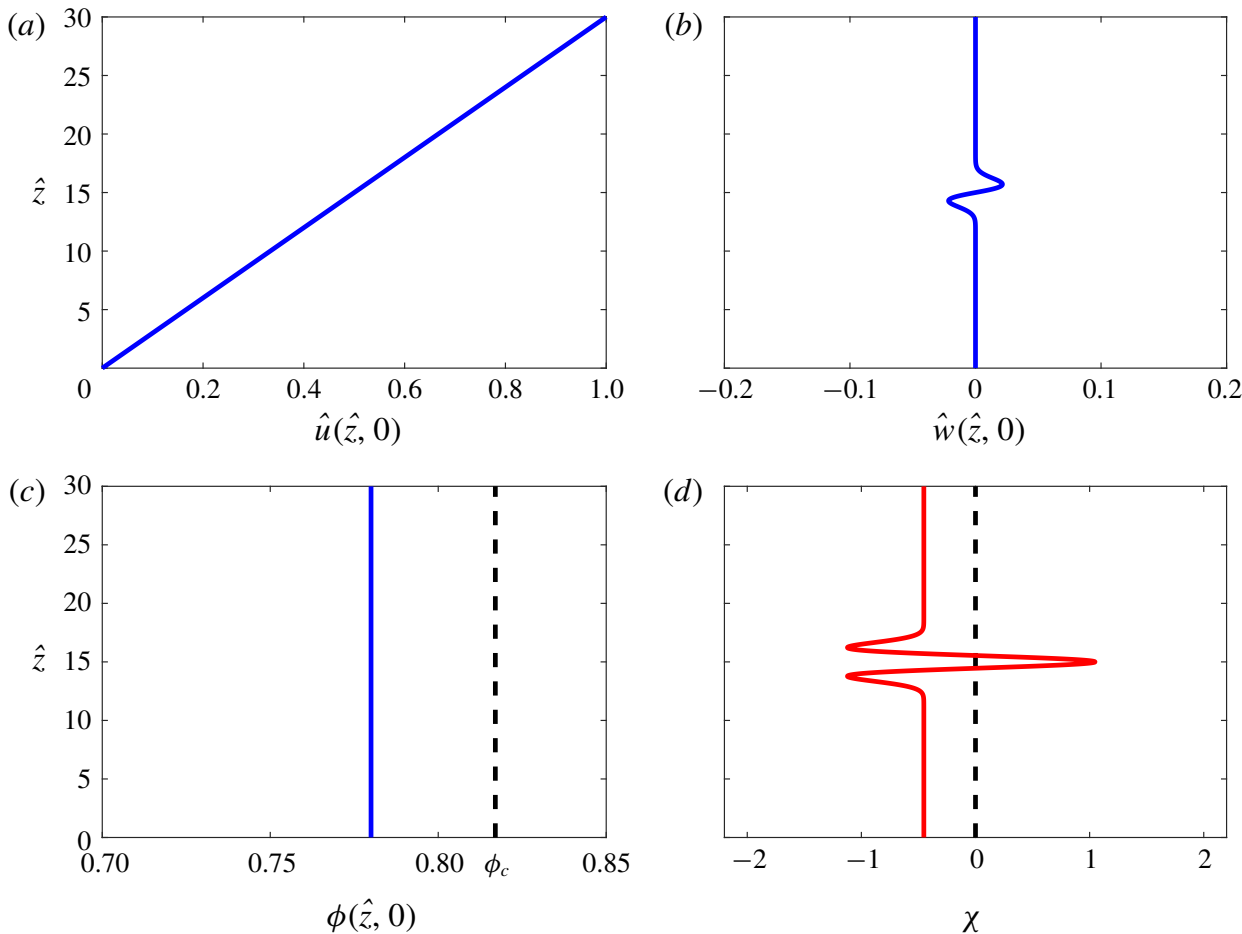

FIGURE 2. Initial conditions used to illustrate the computational difficulties encountered with the $\mu(I), \Phi(I)$-rheology. Panels $(a-c)$ show the non-dimensional velocity components $\hat{u}(\hat{z}, 0), \hat{w}(\hat{z}, 0)$ and the packing fraction $\phi(\hat{z}, 0)$ as a function of the non-dimensional vertical coordinate $\hat{z}$, respectively, for the initial condition (3.16) with $\phi_{0}=0.78, \epsilon=0.05$ and $\hat{H}=30$. Panel $(d)$ shows a plot of the function $\chi(\hat{z})$, defined in (A 18), which determines where the flow is well-posed $(\chi<0)$ or ill-posed $(\chi>0)$.

scheme (see figure 4a).) This is exactly the behaviour that one might expect of a wellposed model, but here it is entirely due to the higher numerical diffusion in the lowresolution scheme. On grid refinement this diffusion is no longer sufficient to suppress the underlying ill-posedness of the equations, and instabilities develop.

For all values of $N_{z}$ above a certain threshold (see figure $4 b$ ), the initial perturbation is amplified indefinitely, causing the method to fail. The time $\hat{t}_{*}$ at which the Chebyshev spectral discretization fails is plotted in figure $4(b)$ as a function of the number of grid points $N_{z}$. Higher spatial resolution computations resolve higher wavenumber instabilities that grow at a faster rate, leading to earlier failure.

\section{Inertial compressible I-dependent rheology (iCIDR)}

The CIDR is a general framework that retains the conservation laws (2.1)-(2.2) and the alignment condition (2.6), but it modifies the other two constitutive equations. The constitutive law for the deviatoric stress (2.7) is replaced by assuming that there is a yield condition such that, if material is deforming, then

$$
\|\boldsymbol{\tau}\|=Y(p, I, \phi)
$$

where $Y$ is called the 'yield function'. In addition, it is assumed that the density evolves dynamically according to a flow rule that is reminiscent of critical state soil 

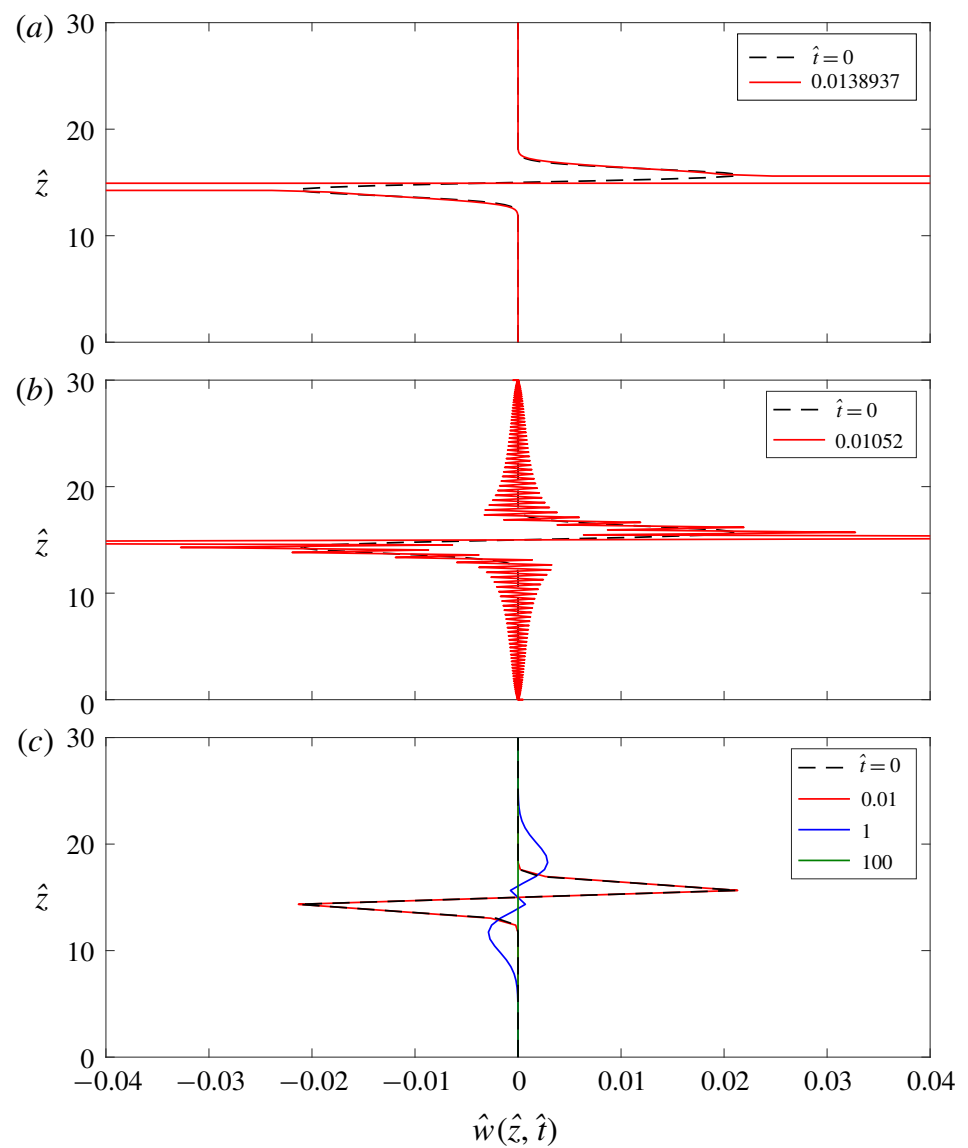

FIGURE 3. Snapshots of the non-dimensional vertical velocity for the $\mu(I), \Phi(I)$-rheology computed with the method of lines in $(a)$ a high-resolution finite difference discretization $\left(N_{z}=201\right)$, (b) a high-resolution Chebychev spectral discretization $\left(N_{z}=201\right)$ and $(c)$ a low-resolution finite difference discretization $\left(N_{z}=47\right)$. In each panel the initial condition of the vertical velocity (3.16) is shown with a dashed black line. Movies 1-3 show the full time evolution of all fields $(\hat{u}, \hat{w}$ and $\phi)$ and are available in the online supplementary material at https://doi.org/10.1017/jfm.2019.476.

mechanics (Schofield \& Wroth 1968; Jackson 1983):

$$
\operatorname{div} \boldsymbol{u}=2 f(p, I, \phi)\|\boldsymbol{S}\|,
$$

where $f$ is called the 'dilatancy function'. Barker et al. (2017) showed that the CIDR equations are linearly well-posed provided the yield and dilatancy functions satisfy the following three conditions:

$$
\begin{gathered}
\frac{\partial Y}{\partial p}-\frac{I}{2 p} \frac{\partial Y}{\partial I}=f+I \frac{\partial f}{\partial I}, \\
\frac{\partial Y}{\partial I}>0 \\
\frac{\partial f}{\partial p}-\frac{I}{2 p} \frac{\partial f}{\partial I}<0 .
\end{gathered}
$$



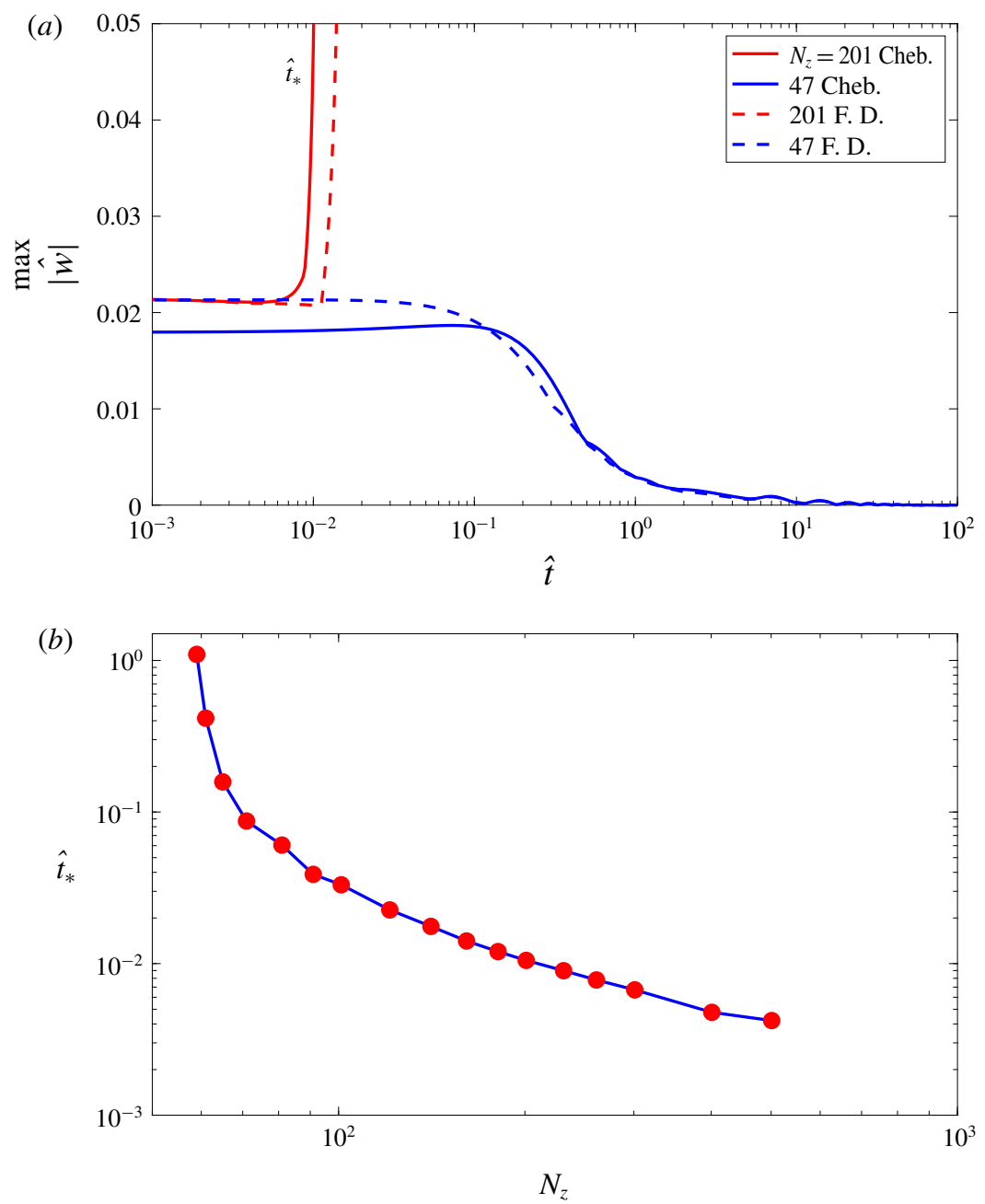

FIGURE 4. (a) A comparison of the temporal evolution of the maximum vertical velocity $|\hat{w}|$ for the numerical computations presented in figure 3. The computations use the method of lines with a finite difference (dashed lines) and a Chebyshev (solids lines) discretization at both high (red lines) and low (blue lines) spatial resolution. The time $\hat{t}_{*}=0.01052$ at which the Chebyshev scheme becomes non-convergent is indicated. $(b)$ The final time $\hat{t}_{*}$ before convergence failure is plotted for the Chebyshev spectral discretization as a function of the number of grid points $N_{z}$. The same plot (not shown) for failure of the finite difference computation is qualitatively similar, but differs in detail.

A key result of this paper is the introduction of new constitutive functions which are motivated by the inertial regime. These are constructed to satisfy both the wellposedness conditions (4.3)-(4.5) and the observed asymptotic steady-state behaviour, known as the $\mu(I)$ and $\Phi(I)$ relations (1.2)-(1.3). These relationships are derived from isochoric (constant volume) flows in steady state (see figure 1 and appendix $C$ ). For the purpose of deriving $Y$ and $f$ they may be conveniently stated as follows:

$$
\text { If } \boldsymbol{\nabla} \cdot \boldsymbol{u}=0, \quad \text { then } f=0, \quad I=\Psi(\phi), \quad \text { and } \quad Y=\mu(\Psi(\phi)) p, \quad(4.6 a-c)
$$




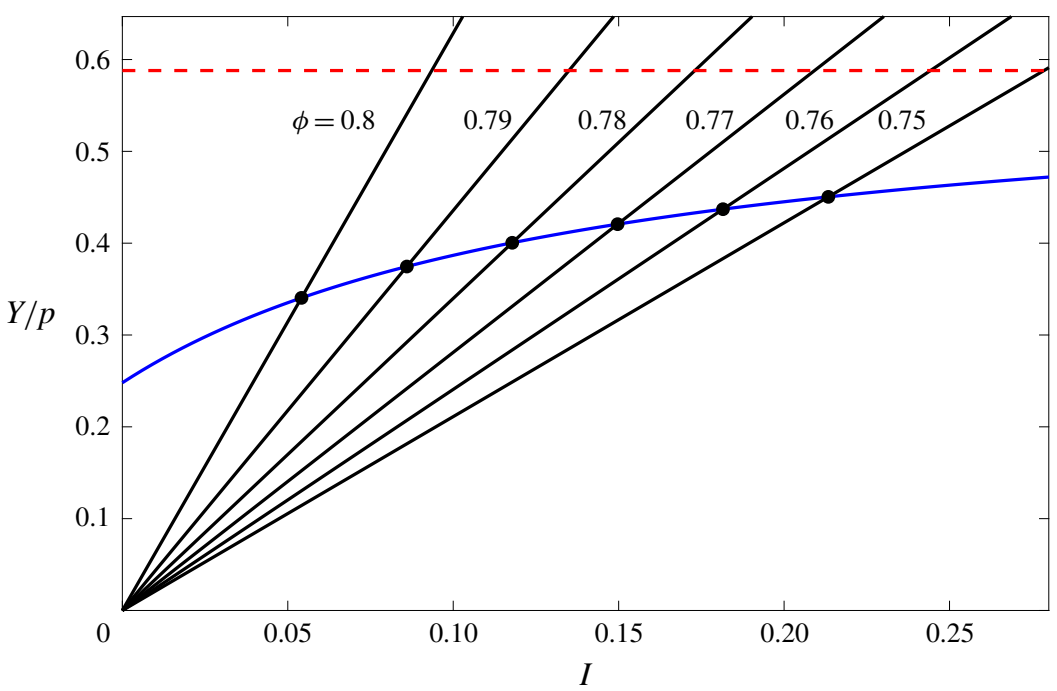

FIgURE 5. Black lines plot the ratio $Y / p$ as a function of $I$ for varying values of the solids volume fraction $\phi$ in iCIDR. The blue line shows $Y / p=\mu(I)$ and the black dots show the steady isochoric state of each of the $Y / p$ lines, i.e. $I=\Psi(\phi)$ and $Y / p=\mu(\Psi(\phi))$. The dashed red line shows the value of $\mu_{d}$.

where $\Psi(\phi)$ is the inverse function of $\Phi(I)$. Even with these constraints there are infinitely many possible choices for the yield function and dilatancy function. What might be described as the simplest acceptable choice for these functions is now proposed.

The starting point for this new theory is the relation $Y=\mu(\Psi(\phi)) p$ in (4.6). However, this cannot lead to a well-posed theory as $\partial_{I} Y=0$ and thus (4.4) is not satisfied. Taking instead the simplest non-trivial $I$-dependence gives

$$
Y(p, I, \phi)=\mu(\Psi(\phi)) \frac{I}{\Psi(\phi)} p,
$$

which reduces to the $\mu(I), \Phi(I)$-rheology when $I=\Psi(\phi)$. Figure 5 is a useful aid for comparing this formula with $\mu(I), \Phi(I)$-rheology. The corresponding dilatancy function is then found through substitution of (4.7) into the well-posedness PDE (4.3). Taking contributions from both the homogeneous and particular solutions of (4.3) and imposing (4.6) gives the expression

$$
f(I, \phi)=\frac{1}{4} \mu(\Psi(\phi))\left[\frac{I}{\Psi(\phi)}-\frac{\Psi(\phi)}{I}\right] .
$$

This theory will be termed 'inertial CIDR' or iCIDR, since, as will be shown, it captures the Bagnold scaling. In addition to satisfying (4.3) and (4.6), it is readily verified that the iCIDR constitutive functions (4.7)-(4.8) satisfy the inequalities (4.4)(4.5). As such, the dynamic equations which result from iCIDR are guaranteed to be linearly well-posed for all deformations and for all values of the solids volume fraction. 
In $\mu(I), \Phi(I)$-rheology $I$ is tied rigidly to $\phi$ through the $I=\Psi(\phi)$ relation (2.12), whereas for iCIDR $I$ evolves through the flow rule (4.2) given the dilatancy function (4.8). Substituting (4.8) into (4.2) yields the quadratic equation

$$
I^{2}-2 \frac{\Psi(\phi) \operatorname{div} \boldsymbol{u}}{\mu(\Psi(\phi))\|\boldsymbol{S}\|} I-\Psi(\phi)^{2}=0
$$

which has the positive root

$$
I=\frac{\Psi(\phi) \operatorname{div} \boldsymbol{u}}{\mu(\Psi(\phi))\|\boldsymbol{S}\|}+\sqrt{\left(\frac{\Psi(\phi) \operatorname{div} \boldsymbol{u}}{\mu(\Psi(\phi))\|\boldsymbol{S}\|}\right)^{2}+\Psi^{2}(\phi)} .
$$

The right-hand side of (4.10) is a function of the solids volume fraction $\phi$ and the ratio $\operatorname{div} \boldsymbol{u} /\|\boldsymbol{S}\|$. Equation (2.9) can be used to determine an equation of state for the pressure

$$
p=\rho_{*}\left(\frac{2 d\|\boldsymbol{S}\|}{I}\right)^{2}
$$

This equation differs from (2.14) only in that $\Psi(\phi)$ is replaced by $I$ defined by (4.10). Since (4.10) depends on the velocity gradient only through the ratio $\operatorname{div} \boldsymbol{u} /\|\boldsymbol{S}\|$, which is unchanged by scaling the velocity gradient, (4.11) satisfies Bagnold scaling (Bagnold 1954).

According to (4.9), if $\|\boldsymbol{S}\| \rightarrow 0$, then $I$ tends to either zero or infinity, depending on the sign of $\operatorname{div} \boldsymbol{u}$. Hence, it seems that the iCIDR constitutive laws may break down if $\|\boldsymbol{S}\|=0$. However, the inertial number $I$ can be bypassed by calculating an alternative expression for the pressure. Substituting (2.9) into (4.9) and solving for $p$ gives

$$
p=\rho_{*}\left(\frac{2 d}{\mu(\Psi(\phi)) \Psi(\phi)}\right)^{2}\left[\sqrt{(\operatorname{div} \boldsymbol{u})^{2}+\mu(\Psi)^{2}\|\boldsymbol{S}\|^{2}}-\operatorname{div} \boldsymbol{u}\right]^{2},
$$

which is well defined for all deformation rates. Note that $p$ is strictly positive unless

$$
\|\boldsymbol{S}\|=0 \quad \text { and } \quad \operatorname{div} \boldsymbol{u} \geqslant 0,
$$

in which case the pressure is zero. Thus, in the absence of shear, there is no pressure if the grains are diverging from one another, but there is a finite, positive pressure if they are converging. Moreover, using the alignment condition (2.6), the yield function (4.7) and the definition of $I$, it follows that the deviatoric stress is given by

$$
\tau_{i j}=\frac{2 d \sqrt{\rho_{*}} \mu(\Psi(\phi))}{\Psi(\phi)} \sqrt{p} S_{i j},
$$

which is also well defined for all deformation rates. Finally, note that if $\phi \rightarrow \phi_{c}$, then $\Psi(\phi) \rightarrow 0$ and the pressure tends to infinity (provided there is some straining).

\section{One-dimensional flow in a shear cell with the iCIDR rheology}

The response of the iCIDR rheology is now tested in the one-dimensional gravitationless shear cell, as studied in $\S 3.2$ for the $\mu(I), \Phi(I)$-rheology. In this geometry the equation of state (4.12) implies the relations for the pressure

$$
p=\rho_{*}\left(\frac{2 d}{\mu(\Psi(\phi)) \Psi(\phi)}\right)^{2}\left[\sqrt{\left(\partial_{z} w\right)^{2}+\mu(\Psi)^{2}\left[\left(\partial_{z} u\right)^{2}+\left(\partial_{z} w\right)^{2}\right]^{2} / 4}-\partial_{z} w\right]^{2},
$$


and the deviatoric stresses (4.14)

$$
\left[\begin{array}{c}
\tau_{x z} \\
\tau_{z z}
\end{array}\right]=\frac{d \sqrt{\rho_{*}} \mu(\Psi(\phi))}{\Psi(\phi)} \sqrt{p}\left[\begin{array}{c}
\partial_{z} u \\
\partial_{z} w
\end{array}\right] .
$$

Substituting these expressions into the conservation laws (3.1)-(3.3) and using the scalings (3.12) implies the non-dimensional iCIDR equations are

$$
\begin{gathered}
\left(\partial_{\hat{t}}+\hat{w} \partial_{\hat{z}}\right) \phi=-\phi \partial_{\hat{z}} \hat{w}, \\
\phi\left(\partial_{\hat{t}}+\hat{w} \partial_{\hat{z}}\right) \hat{u}=\partial_{\hat{z}}\left(\frac{\mu(\Psi(\phi))}{\Psi(\phi)} \sqrt{\tilde{p}} \partial_{\hat{z}} \hat{u}\right), \\
\phi\left(\partial_{\hat{t}}+\hat{w} \partial_{\hat{z}}\right) \hat{w}=-\partial_{\hat{z}} \tilde{p}+\partial_{\hat{z}}\left(\frac{\mu(\Psi(\phi))}{\Psi(\phi)} \sqrt{\tilde{p}} \partial_{\hat{z}} \hat{w}\right),
\end{gathered}
$$

where the pressure is given by

$$
\tilde{p}=\left(\frac{2}{\mu(\Psi(\phi)) \Psi(\phi)}\right)^{2}\left[\sqrt{\left(\partial_{\hat{z}} \hat{w}\right)^{2}+\mu(\Psi)^{2}\left[\left(\partial_{\hat{z}} \hat{u}\right)^{2}+\left(\partial_{\hat{z}} \hat{w}\right)^{2}\right]^{2} / 4}-\partial_{\hat{z}} \hat{w}\right]^{2} .
$$

The pressure (5.6) can be substituted directly into (5.4)-(5.5) to produce a system of three equations for $\phi, \hat{u}$ and $\hat{w}$. Unlike the incompressible $\mu(I)$-rheology and the incompressible Navier-Stokes equations, in which pressure must be determined globally as part of solving the equations, all terms in this system (5.4)-(5.5) are explicitly specified locally. This makes the development of numerical methods much simpler.

Figure 6(a) and supplementary movies 4 and 5 show numerical solutions according to iCIDR for the one-dimensional gravity-free flow of $\S 3$, specifically for initial conditions (3.16). Note that the solution converges smoothly to steady state, the same steady state as for the $\mu(I), \Phi(I)$-rheology (3.10), without any sign of the catastrophic resolution-dependent blow-up seen before. The simulations are computed using the method of lines with the finite difference discretization for both $N_{z}=47$ and 201 grid points. Note that the solutions in figure $6(a)$ lie directly on top of one another! This shows that these solutions are grid converged.

The fact that the iCIDR equations are mathematically well-posed suggests that they can handle larger perturbations from steady state than (3.16). An interesting test case is an initial condition with $\left|\partial_{\hat{z}} \hat{u}\right|=0$ for at least one point. This is interesting because the function $\chi$, defined in (A 18), is infinite when $\left|\partial_{\hat{z}} \hat{u}\right|=0$, so the $\mu(I), \Phi(I)$-rheology is strongly unstable even at low resolution. Following this idea, the results of a simulation with the initial condition

$$
\phi(\hat{z}, 0)=\phi_{0}, \quad \hat{u}(\hat{z}, 0)=\frac{\hat{z}}{\hat{H}}+\hat{a} \sin \left(2 \pi \frac{\hat{z}}{\hat{H}}\right), \quad \hat{w}(\hat{z}, 0)=-\hat{b} \sin \left(2 \pi \frac{\hat{z}}{\hat{H}}\right)
$$

are plotted in figure 7. As figure $7(d)$ shows, with the parameters $\phi_{0}=0.78, \hat{a}=$ 0.16 and $\hat{b}=0.1$, there is a large central region where $\chi>0$ and two points where $\chi$ is infinite. This problem is therefore very strongly ill-posed for the $\mu(I), \Phi(I)$ rheology. Ill-posed behaviour has been verified numerically; indeed, for these initial conditions, even for the coarse grid with $N_{z}=47$, the solution blows up (not shown). By contrast, with the iCIDR equations (5.3)-(5.6) there is no catastrophic blow-up and the simulations smoothly evolve from the initial conditions towards the steady-state 

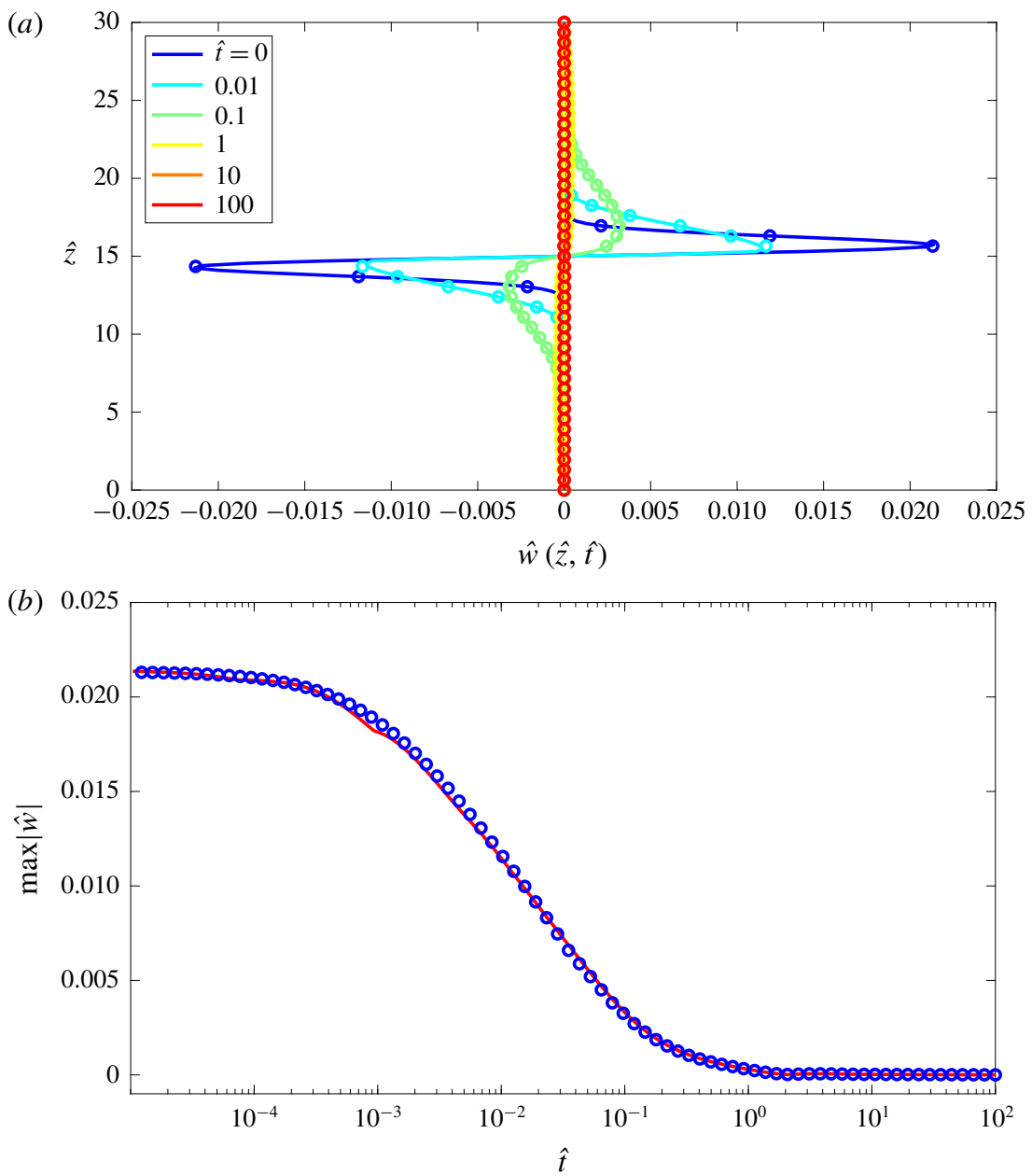

FIgURE 6. (a) Numerical computations for the evolution of the one-dimensional vertical velocity $\hat{w}(\hat{z}, \hat{t})$ for iCIDR. The initial conditions are given by (3.16) with $\phi_{0}=0.78, \epsilon=$ 0.05 and $\hat{H}=30$. The method of lines and first-order finite difference approximations are used to compute the solution, which is shown at a sequence of times with $N_{z}=47$ grid points (open circles) and $N_{z}=201$ grid points (solid lines). This explicitly demonstrates that the numerical solution is grid converged. $(b)$ The evolution of the maximum vertical velocity in time for $N_{z}=47$ grid points (blue circles) and $N_{z}=201$ grid points (red solid line). The numerical solution is very close to the steady-state solution (3.10) by 10 nondimensional units. Supplementary movies 4 and 5 show the evolution of $\phi(\hat{z}, \hat{t}), \hat{u}(\hat{z}, \hat{t})$ and $\hat{w}(\hat{z}, \hat{t})$ for both the finite difference and Chebyshev spectral discretizations.

solution (3.10) as shown in supplementary movie 6 and in figure $7(a-c)$ for both highand low-resolution runs. This convergence is also exhibited in figure $7(e)$ where the maximum vertical velocity is plotted as a function of time: note that the graphs for different resolutions lie on top of one another.

It is interesting to examine the structure of the solution in figure 7. Initially there is a rapid readjustment of the two velocity components with a time constant of the 
(a)

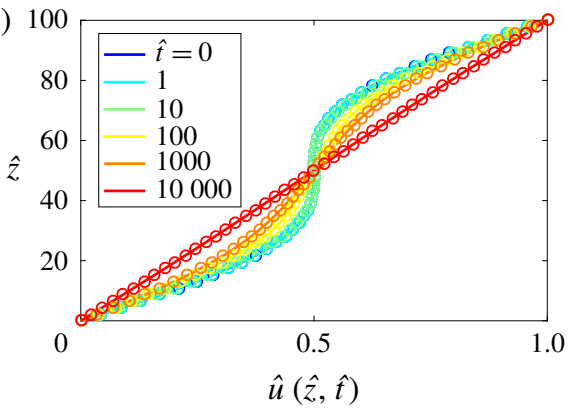

(c)

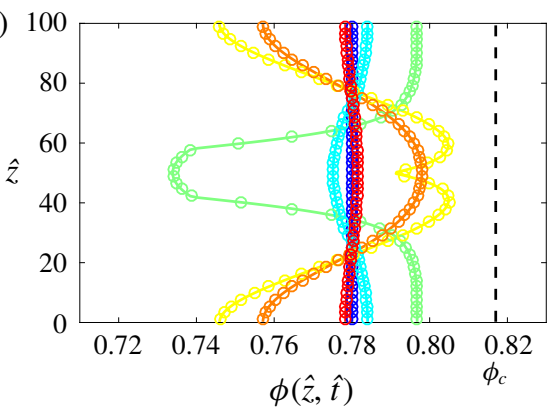

(b)

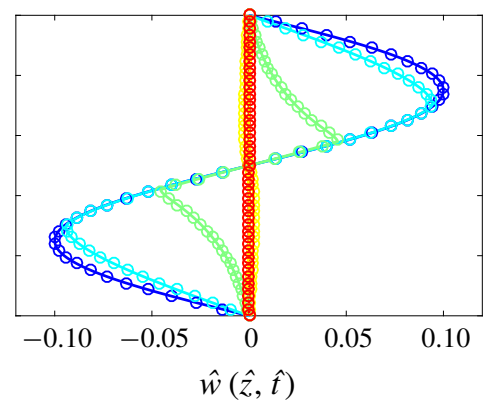

(d)

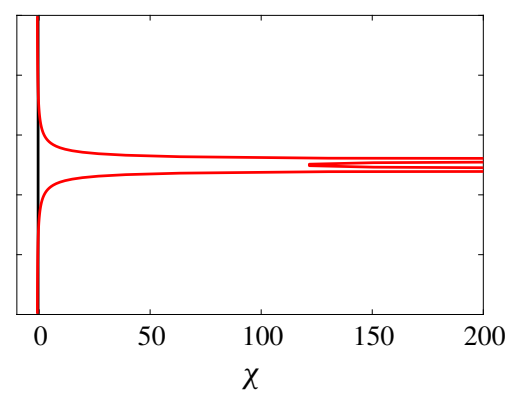

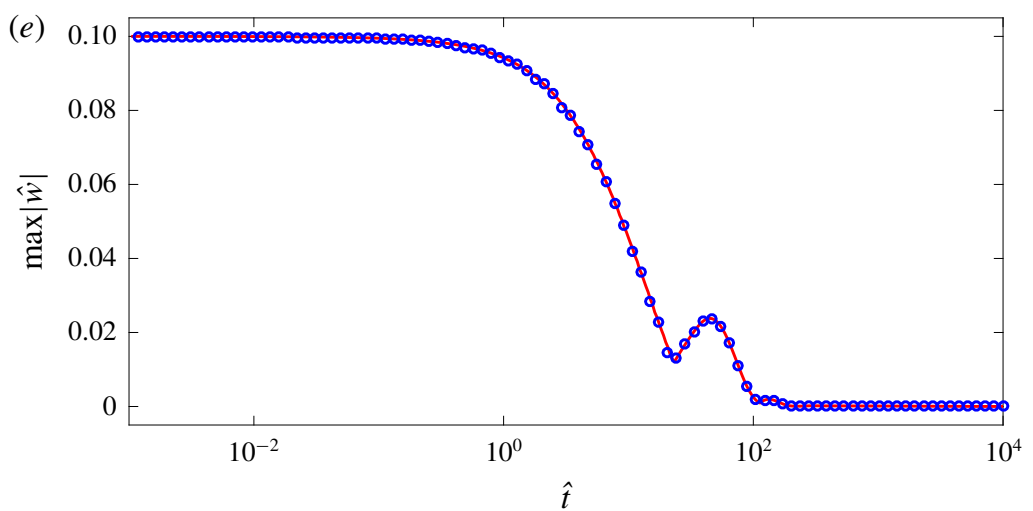

FIgURE 7. Time-dependent numerical simulation for iCIDR. The flow starts from the perturbed initial conditions (5.7) with $\phi_{0}=0.78, \hat{a}=0.16$ and $\hat{b}=0.1$ and converges towards the steady solution (3.10). In panels $(a-c)$ the solid lines correspond to a high spatial resolution $\left(N_{z}=201\right)$ and open circles to a lower resolution $\left(N_{z}=47\right)$. Panel $(d)$ is a plot of $\chi$ defined in (A 18) at $t=0$, which implies that, unlike iCIDR, the $\mu(I), \Phi(I)$-rheology would be strongly ill-posed for these initial conditions. $(e)$ The iCIDR constitutive relations ensure that at both low (open circles, $N_{z}=47$ ) and high (solid red line, $N_{z}=201$ ) spatial resolution the maximum vertical velocity $\hat{w}$ decays to the steady-state value $\hat{w}=0$ everywhere. An animation of the simulation (supplementary movie 6) is available in the online supplementary material.

order of one time unit. The solids volume fraction starts out independent of $\hat{z}$, but it develops a non-trivial profile which changes sign and then decays much more slowly. The latter evolution is responsible for the local maximum of $|\hat{w}|$ near $\hat{t}=3$ shown in figure $7(e)$. 


\section{Comparison of iCIDR with DEM simulations}

The DEM calculations detailed in appendix $\mathrm{C}$ and figure 1, which were used to recover the steady $\mu(I)$ and $\Phi(I)$ relations, are also capable of verifying the time-dependent characteristics of the iCIDR solutions. Here new DEM simulations are initialized with the same procedure that was used to obtain the steady linear shearing solution (3.10), but then the velocity fields are replaced with the sinusoidally perturbed profiles (5.7) discussed in the previous section. As expected, this results in a decay from these applied fields back towards the steady solution. Figure 8 shows that the iCIDR solutions differ by less than $2.5 \%$ relative error from the DEM simulations throughout the dynamics. Figure $8(a-c)$ shows that iCIDR captures the spatial variation of the three flow variables $\phi, \hat{u}$ and $\hat{w}$. Figure $8(d)$ indicates that it also captures complex details of the time evolution. Although more testing is needed, this agreement indicates that iCIDR correctly represents significant aspects of the rheology.

\section{Discussion of related issues}

\subsection{Remarks on ill-posedness}

Issues of ill-posedness in $\mu(I)$-rheology were first raised in Barker et al. (2015). It was shown that, although the dynamic equations derived from incompressible $\mu(I)$ rheology are mathematically well-posed for a large range of inertial numbers, the system is ill-posed when $I$ is too high or too low. The following remarks may help reconcile this ill-posedness with the fact that problems of practical interest (e.g. Lagrée et al. 2011; Staron et al. 2012) have apparently been successfully solved numerically using $\mu(I)$-rheology.

In the first place, ill-posedness effects may be masked in simulations performed on a low-resolution grid, as in $\S 4$ of this paper. Specifically, numerical diffusion may be sufficient to suppress the instability. Ill-posedness may become apparent only through careful comparison of progressively mesh-refined simulations - see figure 4 of Barker \& Gray (2017) and figure 21 of Martin et al. (2017); in these papers certain spurious flow features continue to become ever more finely scaled as the grid size gets smaller. It is sometimes suggested that low-resolution solutions of an ill-posed model might be sufficient for some practical purposes. However, such approaches are scientifically flawed, because the results rely on numerical diffusion to regularize the problem, which is dependent on both grid size and numerical scheme, and is often not known precisely. In our view it is far better to try to understand what physics is missing in the model, and only compute solutions when a well-posed theory has been formulated.

Other issues are the following. (i) In some problems, such as column collapse (Lagrée et al. 2011), the ill-posed region of parameter space may only be active for a short period of time. In such cases careful comparison of numerical results at different spatial resolutions, including some very fine grids, may be needed for non-convergence to become apparent (Barker et al. 2015; Barker \& Gray 2017; Martin et al. 2017). (ii) Ill-posedness may also be partially suppressed by attempts to remove the singularity in the viscosity at low strain rates. Many numerical codes do this by introducing an upper bound on the viscosity, which implies that the material reverts to a Newtonian fluid for slow flows. However, these procedures are ad hoc in nature, and there is no guarantee that ill-posedness is suppressed completely.

In this subsection attention has been focused on the consequences of ill-posedness that may be seen through computations. However, on the mathematical side, it 
(a)

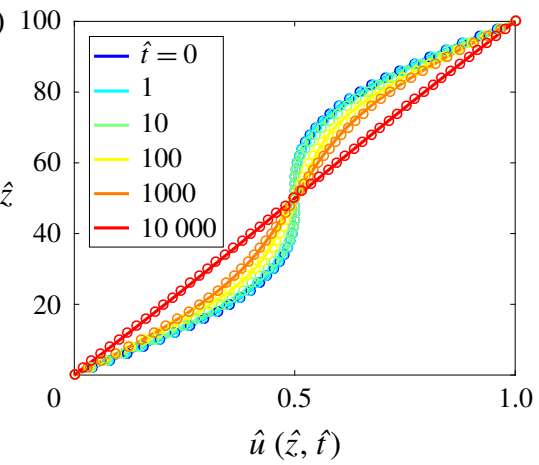

(b)

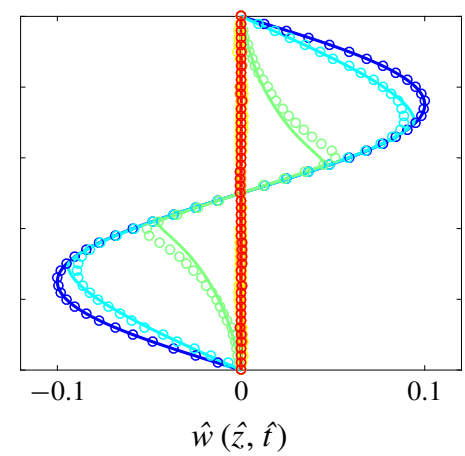

(c)
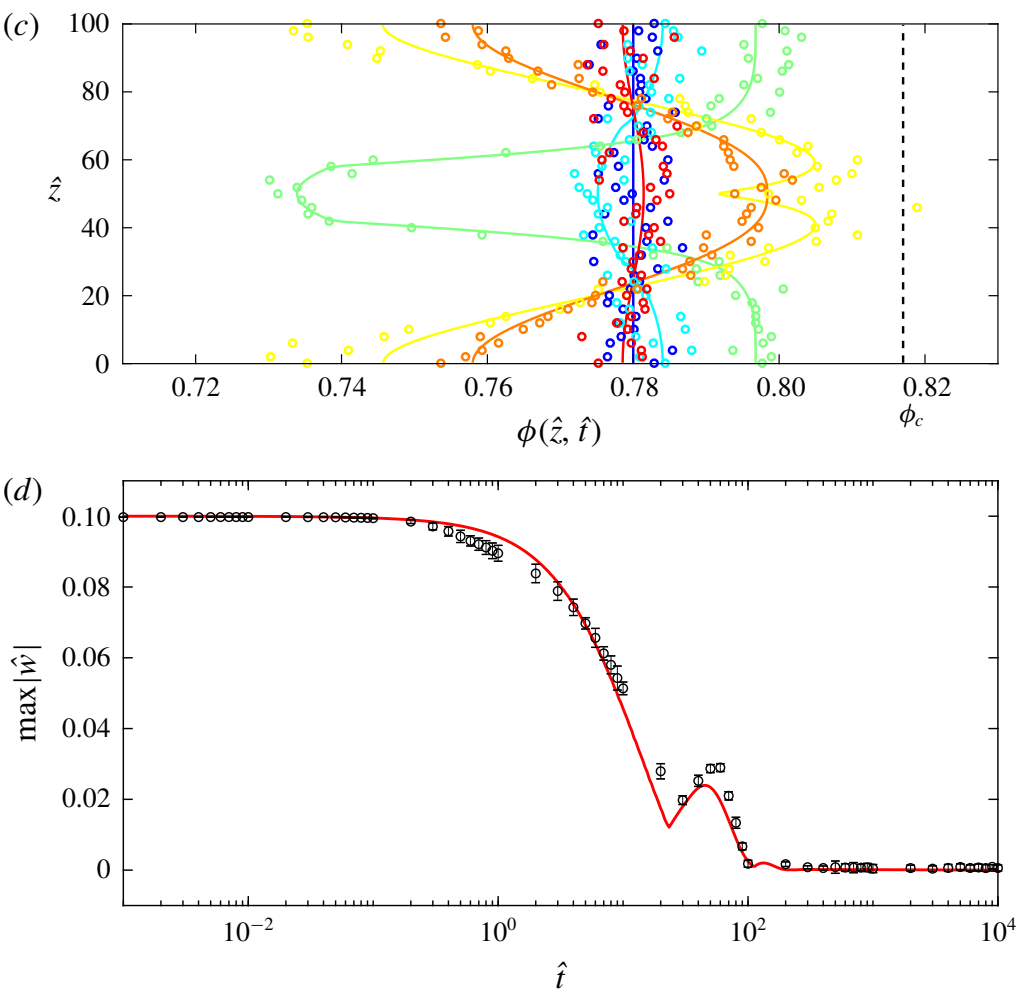

FIGURE 8. A comparison between the iCIDR continuum calculation, detailed in figure 7 , and the results of two-dimensional DEM simulations initialized with the same flow fields. Circles are the mean values and error bars represent the standard deviation of 10 repeat DEM simulations. Supplementary movies 7 and 8 show the evolution of the coarse-grained velocity components $\hat{u}$ and $\hat{w}$ alongside renderings of the particles during the simulation. Note that $\hat{u}$ obtained by DEM simulations is given by the relative velocity between the bottom $(\hat{z}=0)$ and top $(\hat{z}=\hat{H})$ layers under the Lees-Edwards boundary conditions in order to simplify the comparison with the iCIDR continuum calculation.

seems likely that solutions of an ill-posed initial-value problem exist only for a very restricted set of initial conditions, far too restricted to model physical problems. A rigorous proof of this assertion for complicated PDEs like iCIDR would be difficult, but for a simpler example let us refer to appendix 2 of Barker et al. (2017) for a 
proof of the following fact: the backwards heat equation

$$
\partial_{t} u=-\partial_{x x} u, \quad u(x, 0)=\epsilon|\sin x|^{p},
$$

where $\epsilon \neq 0$, cannot be solved on any non-zero interval $\left\{0<t<t_{0}\right\}$ unless $p$ is an even non-negative integer. The singularities of the initial conditions at $x=n \pi, n=$ $0, \pm 1, \pm 2, \ldots$ (which are extremely mild if $p$ is large) make such solutions impossible.

\subsection{Behaviour at the boundary of and outside the inertial regime}

Regarding the boundary of the inertial regime, note in (2.14) and (5.11) that $p$ tends to infinity as $\phi \rightarrow \phi_{c}$. In DEM simulations, Chialvo et al. (2012) found that this growth was cut off and blended into the pressure from the intermediate regime through the formula

$$
p_{\text {blend }}=\frac{p_{\text {inert }} p_{\text {itm }}}{p_{\text {inert }}+p_{\text {itm }}},
$$

where

$$
p_{i t m}=\alpha_{i t m}\left(\frac{k}{d}\right)^{3 / 4}\left(\sqrt{\rho_{*}} d \dot{\gamma}\right)^{1 / 2} .
$$

Thus the pressure tends to $p_{i t m}$ as $\phi \rightarrow \phi_{c}$, where the limit pressure depends on the elastic modulus. Although it is beyond the scope of the present paper, it is anticipated that a more complete version of CIDR would remain valid across regime boundaries.

Granular flow at densities $\phi>\phi_{c}$ (provided the strain rate is not too large) corresponds to what is called the quasi-static regime (Otsuki \& Hayakawa 2009; Chialvo et al. 2012; Singh et al. 2015). In this regime stresses may remain non-zero even as the strain rate tends to zero, i.e. a static yield stress exists; the scale of these static stresses is set by $k / d$, where $k$ is the spring constant in DEM simulations.

CIDR is a general theory that can model granular material outside, as well as inside, of the inertial regime. In particular, the version of CIDR in $\S 2$ (e) of Barker et al. (2017), which was motivated by critical state soil mechanics (Schofield \& Wroth 1968), has a non-zero static yield stress, as in the quasi-static regime in DEM simulations. (In that paper, to facilitate comparison with Silbert et al. (2001), the stress scale was chosen as $\rho_{*} g d$, i.e. dependent on gravitational acceleration $g$. This was an unfortunate choice with no intrinsic significance. A more appropriate choice would have been $k / d$, where $k$ is the spring constant in DEM simulations.)

\subsection{Extensions to the theory}

Inertial CIDR is also able to incorporate non-monotonicity of the $\mu(I)$ function (DeGiuli \& Wyart 2017), which is crucial for modelling hysteretic effects, such as coexisting static and moving regions, in depth-averaged avalanche models (Daerr \& Douady 1999; Pouliquen \& Forterre 2002; Edwards \& Gray 2015; Edwards et al. 2017; Russell et al. 2019). Non-monotonic $\mu=\mu(I)$ functions are problematic in the incompressible $\mu(I)$-rheology, because they imply that the theory is always ill-posed in regions of decreasing friction (Barker et al. 2015). For iCIDR, however, having a non-monotonic $\mu=\mu(I)$ function is not a problem, because it is formulated in terms of the solids volume fraction, i.e. $\mu=\mu(\Psi(\phi))$, and so it does not affect the well-posedness conditions (4.3)-(4.5).

At present iCIDR is explicitly a local theory which cannot account for the observed role of fluctuations that inspired the non-local theories of Pouliquen \& Forterre (2009), Kamrin \& Koval (2012) and Bouzid et al. (2013). Inclusion of these effects is an important direction for future work. 


\section{Conclusions}

The iCIDR equations, introduced in this paper, provide a continuum model for fluid-like inertial flows of rigid spherical particles that lie below a critical volume fraction, above which the compressibility of the grains becomes important (Otsuki \& Hayakawa 2009; Chialvo et al. 2012). One striking aspect of the iCIDR theory is its simplicity: it is a minimal extension of $\mu(I), \Phi(I)$-rheology with no extra variables, no extra parameters, no extra evolution equations beyond conservation of mass and momentum and no extra boundary conditions. While retaining the success of $\mu(I), \Phi(I)$-rheology for steady inertial flow, iCIDR is well-posed, thermodynamically sound (Onsager symmetric and dissipative) and agrees well with transient DEM simulations in a one-dimensional gravity-free shear cell.

Inertial CIDR is very well suited to numerical calculations because the pressure is defined by a local equation of state. This contrasts with incompressible theories, in which the pressure can only be found globally as part of solving the equations. The numerical simulations presented in this paper are therefore a particularly encouraging proof of concept and it is hoped that other existing numerical methods can similarly be modified in order to bring the advantages of the iCIDR formulation to a wide range of practical applications.

\section{Acknowledgements}

The research of M.S. is supported by National Science Foundation grant DMS-1812445. This research was also supported by NERC grants NE/E003206/1 and NE/K003011/1 as well as EPSRC grants EP/I019189/1, EP/K00428X/1 and EP/M022447/1. J.M.N.T.G. is a Royal Society Wolfson Research Merit Award holder (WM150058) and an EPSRC Established Career Fellow (EP/M022447/1). All research data supporting this publication are directly available within the article.

\section{Supplementary movies}

Supplementary movies are available at https://doi.org/10.1017/jfm.2019.476.

\section{Appendix A. Ill-posedness of the one-dimensional $\mu(I), \Phi(I)$-rheology}

Although there are some technical complications, effectively the dynamic equations of $\mu(I), \Phi(I)$-rheology for two-dimensional flow are always ill-posed (Heyman et al. 2017). As in Barker et al. (2015), the ill-posedness has a directional character: plane-wave solutions (in all space) in certain directions suffer uncontrolled growth while those in other directions decay smoothly to uniform flow. If, as in $\S 3$, solutions depending on only one spatial variable are sought, these one-dimensional equations may be either well-posed or ill-posed, depending on whether the one independent direction retained corresponds to one of the stable or unstable directions. In this appendix, conditions for the ill-posedness of the one-dimensional system (3.1)-(3.8) are derived.

The equations (3.1)-(3.3) together with (3.6) and (3.8) are linearized around a base flow $\left(\phi^{0}, u^{0}, w^{0}\right)$ in the dependent variables $(\phi, u, w)$ by introducing perturbations $(\breve{\phi}, \breve{u}, \breve{w})$ of the form

$$
\left[\begin{array}{l}
\phi \\
u \\
w
\end{array}\right]=\left[\begin{array}{l}
\phi^{0}(z, t) \\
u^{0}(z, t) \\
w^{0}(z, t)
\end{array}\right]+\left[\begin{array}{l}
\breve{\phi}(z, t) \\
\breve{u}(z, t) \\
\breve{w}(z, t)
\end{array}\right],
$$


and then freezing the base flow coefficients. In the linearization the highest-order derivatives of the perturbed variables are retained in each equation, together with the convective derivatives. This leads to linearized equations of the form

$$
\partial_{t}\left[\begin{array}{c}
\breve{\phi} \\
\breve{u} \\
\breve{w}
\end{array}\right]=\left[\begin{array}{ccc}
-w^{0} \partial_{z} & 0 & -\phi^{0} \partial_{z} \\
a_{1} \partial_{z} & b_{11} \partial_{z z}-w^{0} \partial_{z} & b_{12} \partial_{z z} \\
a_{2} \partial_{z} & b_{21} \partial_{z z} & b_{22} \partial_{z z}-w^{0} \partial_{z}
\end{array}\right]\left[\begin{array}{c}
\breve{\phi} \\
\breve{u} \\
\breve{w}
\end{array}\right],
$$

where $a_{i}$ and $b_{i j}$ are constant coefficients derived from the pressure (3.6) and the deviatoric stresses (3.8).

The linearized system (A 2) admits normal-mode solutions of the form

$$
\left[\begin{array}{l}
\breve{\phi}(z, t) \\
\breve{u}(z, t) \\
\breve{w}(z, t)
\end{array}\right]=\mathrm{e}^{\mathrm{i} \xi z+\lambda t}\left[\begin{array}{l}
\tilde{\phi} \\
\tilde{u} \\
\tilde{w}
\end{array}\right]
$$

in which $\xi$ is the wavenumber, $\lambda$ is the temporal growth rate and $\tilde{\phi}, \tilde{u}$ and $\tilde{w}$ are constants. Substituting these into (A2) reveals that $\lambda$ must be an eigenvalue of the matrix

$$
\boldsymbol{A}(\xi)=\left[\begin{array}{ccc}
-\mathrm{i} w^{0} \xi & 0 & -\mathrm{i} \phi^{0} \xi \\
\mathrm{i} a_{1} \xi & -b_{11} \xi^{2}-\mathrm{i} w^{0} \xi & -b_{12} \xi^{2} \\
\mathrm{i} a_{2} \xi & -b_{21} \xi^{2} & -b_{22} \xi^{2}-\mathrm{i} w^{0} \xi
\end{array}\right] .
$$

The imaginary terms $-\mathrm{i} w^{0} \xi$ on the diagonal shift the eigenvalues $\lambda$, but do not affect stability or well-posedness, which are governed by the real part of $\lambda$. The vertical component of the base state velocity $w^{0}$ is therefore set to zero in what follows.

Denoting the bottom right $2 \times 2$ matrix of terms multiplying $-\xi^{2}$ as

$$
\boldsymbol{\Gamma}=\left[\begin{array}{ll}
b_{11} & b_{12} \\
b_{21} & b_{22}
\end{array}\right],
$$

the eigenvalues are calculated by solving

$$
\operatorname{det}(\boldsymbol{A}(\xi)-\lambda \boldsymbol{I})=-\left(\lambda^{3}+A \lambda^{2}+B \lambda+C\right)=0,
$$

where the coefficients are

$$
A=(\operatorname{tr} \boldsymbol{\Gamma}) \xi^{2}+O(\xi), \quad B=(\operatorname{det} \boldsymbol{\Gamma}) \xi^{4}+O\left(\xi^{3}\right), \quad C=O\left(\xi^{4}\right) . \quad(\mathrm{A} 7 a-c)
$$

To test for ill-posedness, the large-wavenumber limit $\xi \rightarrow \infty$ is taken. Balancing the order of terms in (A 6), it is clear that $\lambda(\xi) \sim \xi^{2}$, so the substitution $\lambda=\Lambda \xi^{2}$ is made. Then the terms of maximal order in (A 6), i.e. $O\left(\xi^{6}\right)$, have the coefficient $\Lambda^{3}+(\operatorname{tr} \boldsymbol{\Gamma}) \Lambda^{2}+(\operatorname{det} \boldsymbol{\Gamma}) \Lambda$. Thus, to leading order, either $\Lambda=0$, or $\Lambda$ is a solution of

$$
\Lambda^{2}+(\operatorname{tr} \boldsymbol{\Gamma}) \Lambda+\operatorname{det} \boldsymbol{\Gamma}=0,
$$

i.e. one of the two roots

$$
\Lambda_{ \pm}=\frac{1}{2}\left[-\operatorname{tr} \boldsymbol{\Gamma} \pm \sqrt{(\operatorname{tr} \boldsymbol{\Gamma})^{2}-4 \operatorname{det} \boldsymbol{\Gamma}}\right]
$$


To determine the signs of these roots, the coefficients in (A 2) are now evaluated. If the superscript 0 notation is dropped, the coefficients in (A 2) are then

$$
\left.\begin{array}{c}
a_{1}=\frac{2 d^{2}}{\phi} \partial_{z} u\|\boldsymbol{S}\| \partial_{\phi}\left(\frac{M(\phi)}{\Psi^{2}(\phi)}\right), \\
a_{2}=\frac{2 d^{2}\|\boldsymbol{S}\|}{\phi}\left[\partial_{z} w \partial_{\phi}\left(\frac{M(\phi)}{\Psi^{2}(\phi)}\right)-2\|\boldsymbol{S}\| \partial_{\phi}\left(\frac{1}{\Psi^{2}(\phi)}\right)\right], \\
b_{11}=\frac{d^{2} M(\phi)}{2 \phi \Psi^{2}(\phi)} \frac{2\left(\partial_{z} u\right)^{2}+\left(\partial_{z} w\right)^{2}}{\|\boldsymbol{S}\|}, \quad b_{12}=\frac{d^{2} M(\phi)}{2 \phi \Psi^{2}(\phi)} \frac{\partial_{z} u \partial_{z} w}{\|\boldsymbol{S}\|}, \\
b_{21}=\frac{d^{2} M(\phi)}{2 \phi \Psi^{2}(\phi)} \frac{\partial_{z} u \partial_{z} w}{\|\boldsymbol{S}\|}-\frac{2 d^{2}}{\phi \Psi^{2}(\phi)} \partial_{z} u, \\
b_{22}=\frac{d^{2} M(\phi)}{2 \phi \Psi^{2}(\phi)} \frac{\left(\partial_{z} u\right)^{2}+2\left(\partial_{z} w\right)^{2}}{\|\boldsymbol{S}\|}-\frac{2 d^{2}}{\phi \Psi^{2}(\phi)} \partial_{z} w,
\end{array}\right\}
$$

where all values are evaluated with the base-state fields

$$
M(\phi)=\mu(\Psi(\phi)) \quad \text { and } \quad\|\boldsymbol{S}\|=\frac{1}{2} \sqrt{\left(\partial_{z} u\right)^{2}+\left(\partial_{z} w\right)^{2}}
$$

From (A 10) and (A 5) it follows that

$$
\operatorname{tr} \boldsymbol{\Gamma}=\frac{d^{2}}{\phi \Psi^{2}}\left[3 M \sqrt{\left(\partial_{z} u\right)^{2}+\left(\partial_{z} w\right)^{2}}-2 \partial_{z} w\right]
$$

and

$$
\operatorname{det} \boldsymbol{\Gamma}=\frac{2 d^{4} M}{\phi^{2} \Psi^{4}} \sqrt{\left(\partial_{z} u\right)^{2}+\left(\partial_{z} w\right)^{2}}\left[M \sqrt{\left(\partial_{z} u\right)^{2}+\left(\partial_{z} w\right)^{2}}-\partial_{z} w\right] .
$$

The two roots in (A 9) are real since

$$
(\operatorname{tr} \boldsymbol{\Gamma})^{2}-4 \operatorname{det} \boldsymbol{\Gamma}=\left(\frac{2 d^{2}}{\phi \Psi^{2}}\right)^{2}\left(M\|\boldsymbol{S}\|-\partial_{z} w\right)^{2} \geqslant 0 .
$$

Since $\lambda_{+} \sim \Lambda_{+} \xi^{2}$ as $\xi \rightarrow \infty$, the system is linearly ill-posed if the larger root $\Lambda_{+}$ is positive, which occurs if either

$$
\text { (a) } \operatorname{tr} \boldsymbol{\Gamma}<0 \text { or } \quad \text { (b) } \operatorname{det} \boldsymbol{\Gamma}<0 \text {. }
$$

It may be seen from (A 12) and (A 13) that if $\operatorname{tr} \boldsymbol{\Gamma}<0$ then $\operatorname{det} \boldsymbol{\Gamma}<0$, so it suffices to consider only case (b). Thus,

$$
\frac{\partial_{z} w}{\sqrt{\left(\partial_{z} u\right)^{2}+\left(\partial_{z} w\right)^{2}}}>M(\phi)
$$

is a sufficient condition for ill-posedness.

Conversely, suppose

$$
\frac{\partial_{z} w}{\sqrt{\left(\partial_{z} u\right)^{2}+\left(\partial_{z} w\right)^{2}}}<M(\phi)
$$


Then $\operatorname{tr} \boldsymbol{\Gamma}$ and $\operatorname{det} \boldsymbol{\Gamma}$ are both positive, so $\Lambda_{ \pm}$are both negative, and hence $\lambda_{ \pm} \sim \Lambda_{ \pm} \xi^{2}$ are bounded above. From (A 4), the third eigenvalue $\lambda_{0}$ asymptotes to $-C / B$ as $\xi \rightarrow$ $\infty$. But $C / B$ is the ratio of two quartics and $B \neq 0$ since $\operatorname{det} \boldsymbol{\Gamma} \neq 0$. Thus $\lambda_{0}$ also remains bounded as $\xi \rightarrow \infty$. In other words, (A 17) is a sufficient condition for wellposedness.

To rewrite (A 16), (A 17) in a way that is useful for the computations in $\S 3.2$, let us define

$$
\chi=\frac{\partial_{z} w}{\left|\partial_{z} u\right|}-\frac{M}{\sqrt{1-M^{2}}} .
$$

The one-dimensional computations with $\mu(I), \Phi(I)$-rheology will be

$$
\text { ill-posed where } \chi>0 \text { and well-posed where } \chi<0 \text {. }
$$

\section{Appendix B. Positive stress power and Onsager symmetry}

Goddard \& Lee (2018) examined equations for granular flow in light of Edelen's (1972) theory of irreversibility. They showed that the model of Heyman et al. (2017), which includes $\mu(I), \Phi(I)$-rheology, is not Onsager symmetric and not even properly dissipative. In this appendix the iCIDR equations are shown to have positive stress power and Onsager symmetry.

Positive-definite stress power means that all deformations with non-zero stress dissipate energy; i.e. in symbols, the dissipation rate $\mathcal{D}$ satisfies

$$
\mathcal{D}=\operatorname{tr}(\boldsymbol{\sigma} \boldsymbol{D})>0 \text { if }\|\boldsymbol{D}\|>0 \text { and }\|\boldsymbol{\sigma}\|>0 .
$$

Using the decompositions of the stress (2.3) and the strain rate (2.5)

$$
\|\boldsymbol{D}\|=\sqrt{\|\boldsymbol{S}\|^{2}+(\operatorname{div} \boldsymbol{u})^{2} / 4}, \quad\|\boldsymbol{\sigma}\|=\sqrt{\|\boldsymbol{\tau}\|^{2}+p^{2}},
$$

and it follows that

$$
\mathcal{D}=\operatorname{tr}(\boldsymbol{\tau} \mathbf{S})-p \operatorname{div} \boldsymbol{u}
$$

Substituting the alignment condition (2.6) and the dilatancy law (4.2) and recalling that $\|\boldsymbol{S}\|^{2}=\operatorname{tr}\left(\boldsymbol{S}^{2}\right) / 2$, implies that

$$
\mathcal{D}=2\|\boldsymbol{\tau}\|\|\boldsymbol{S}\|-2 f\|\boldsymbol{S}\| p
$$

The yield condition (4.1), the yield function (4.7) and the dilatancy function (4.8) then imply that the dissipation rate is

$$
\mathcal{D}=M\|\boldsymbol{S}\|\left[\frac{3}{2} \frac{I}{\Psi}+\frac{1}{2} \frac{\Psi}{I}\right] p,
$$

where $M=\mu(\Psi(\phi))$ and $\Psi=\Psi(\phi)$. Thus, the dissipation rate is non-negative and in fact positive if $\|\boldsymbol{S}\|>0$ (and hence, by (4.12), $p>0$ ). To determine what happens when $\|\boldsymbol{S}\|=0$, the definition of the inertial number (2.9) is substituted into (B 5) to show that

$$
\mathcal{D}=\frac{3 d M \sqrt{\rho_{*}}}{\Psi}\|\boldsymbol{S}\|^{2} \sqrt{p}+\frac{M \Psi}{4 d \sqrt{\rho_{*}}} p^{3 / 2}
$$

As before, of course, $\mathcal{D}>0$ if $\|\boldsymbol{S}\|>0$. If $\|\boldsymbol{S}\|=0$ but $\operatorname{div} \boldsymbol{u}<0$, then by (4.12) the second term in (B 6) is positive. If $\|\boldsymbol{S}\|=0$ but $\operatorname{div} \boldsymbol{u}>0$, then indeed $\mathcal{D}=0$, but $p=0$ 
and by (4.14) $\|\boldsymbol{\tau}\|=0$, so $\|\boldsymbol{\sigma}\|=0$. This completes the verification of (B 1), i.e. the iCIDR equations always dissipate energy provided there is some deformation and the stresses are non-zero.

Since the constitutive laws have positive stress power, it follows from Edelen (1972) that (i) all deformations are irreversible and increase entropy, (ii) the iCIDR constitutive laws satisfy Onsager symmetry and (iii) each component $\sigma_{i j}$ of the stress equals the derivative of a convex dissipation potential $\psi(\boldsymbol{D})$ with respect to $D_{i j}$. Regarding point (iii), it is convenient to derive $\psi$ through its Legendre transform,

$$
\varphi(\mathbf{S}, p)=\inf _{\delta} \psi(\boldsymbol{S}, \delta)+p \delta
$$

where $\delta$ is a shorter notation for $\operatorname{div} \boldsymbol{u}$ when it appears as a dummy variable. Consider the function

$$
\varphi(\boldsymbol{S}, p)=\frac{2 d \sqrt{\rho_{*}} M}{\Psi}\|\boldsymbol{S}\|^{2} \sqrt{p}-\frac{M \Psi}{6 d \sqrt{\rho_{*}}} p^{3 / 2} .
$$

The claim is that the iCIDR constitutive relations can be expressed as the derivatives

$$
\text { (a) } \tau_{i j}=\frac{\partial \varphi}{\partial S_{i j}} \quad \text { and } \quad \text { (b) } \operatorname{div} \boldsymbol{u}=\frac{\partial \varphi}{\partial p} \text {. }
$$

The first relation implies

$$
\frac{\partial \varphi}{\partial S_{i j}}=\frac{2 d \sqrt{\rho_{*}} M}{\Psi} S_{i j} \sqrt{p}=\frac{M}{\Psi} \frac{2 d\|\boldsymbol{S}\|}{\sqrt{p / \rho_{*}}} \frac{S_{i j}}{\|\boldsymbol{S}\|} p,
$$

which, in light of (2.6), (4.1) and (4.7), verifies (B 9a). Whilst the the second implies

$$
\frac{\partial \varphi}{\partial p}=\frac{d \sqrt{\rho_{*}} M}{\Psi}\|\boldsymbol{S}\|^{2} \frac{1}{\sqrt{p}}-\frac{M \Psi}{4 d \sqrt{\rho_{*}}} \sqrt{p}=\frac{M\|\boldsymbol{S}\|}{2}\left[\frac{1}{\Psi} \frac{2 d\|\boldsymbol{S}\|}{\sqrt{p / \rho_{*}}}-\Psi \frac{\sqrt{p / \rho_{*}}}{2 d\|\boldsymbol{S}\|}\right],
$$

which, in light of (4.2) and (4.8), verifies (B 9b). The dissipation potential $\psi(\boldsymbol{D})$ is the inverse Legendre transform of $\varphi$, which may be written

$$
\psi(\boldsymbol{S}, \operatorname{div} \boldsymbol{u})=\varphi(\boldsymbol{S}, P(\boldsymbol{S}, \operatorname{div} \boldsymbol{u}))-P(\boldsymbol{S}, \operatorname{div} \boldsymbol{u}) \operatorname{div} \boldsymbol{u},
$$

where $P(\boldsymbol{S}, \operatorname{div} \boldsymbol{u})$ is the pressure given by (4.12). Mixing stress and strain-rate variables, $\psi$ can be calculated as a function of $S$ and $p$ by replacing $P$ in (B 12) by $p$ and substituting (B 11) for $\operatorname{div} \boldsymbol{u}$, which yields

$$
\psi=\frac{d M \sqrt{\rho_{*}}}{\Psi}\|\boldsymbol{S}\|^{2} \sqrt{p}+\frac{M \Psi}{12 d \sqrt{\rho_{*}}} p^{3 / 2} .
$$

Observe that

$$
\psi=\mathcal{D} / 3
$$

a result that follows from the formula (Goddard \& Lee 2018)

$$
\psi(\boldsymbol{D})=\int_{0}^{1} \mathcal{D}(\lambda \boldsymbol{D}) \frac{\mathrm{d} \lambda}{\lambda}
$$

and the observation that $\mathcal{D}$ is homogeneous in $\boldsymbol{D}$ of degree 3 . It is not proved directly that $\psi$ is convex, but rather the fact that the iCIDR constitutive relations are wellposed (Barker et al. 2017) is used to deduce this (Goddard \& Lee 2018). 


\section{Appendix C. Details of the DEM calculations}

Two-dimensional DEM simulations (Cundall \& Strack 1979) were performed in a shear-box geometry, both to confirm the well-established steady behaviour and to explore transient flows. The domain is a rectangle in $(x, z)$ space, with all units non-dimensionalized with the scalings (3.12), such that $0 \leqslant \hat{x}<\hat{L}$ and $0 \leqslant \hat{z}<\hat{H}$. Boundary conditions and the system size are then designed to suppress confinement effects so that the volume can be taken to be a representative bulk element. Periodicity is enforced in the $\hat{x}$ direction and Lees-Edwards boundary conditions (Lees \& Edwards 1972) are applied at the bottom $\hat{z}=0$ and top $\hat{z}=\hat{H}$ of the domain. There is no gravity applied to the system and the only driving is provided by the difference in horizontal velocity between the top and bottom $V_{0}$, as prescribed by the Lees-Edwards algorithm.

Details of the precise DEM simulation algorithm can be found in Otsuki \& Hayakawa (2011) as an identical method is employed here. Normal forces $\boldsymbol{f}^{(n)}$ between particles are calculated from a linear spring-dashpot arrangement with an associated spring constant $k^{(n)}$ and viscous dissipation constant $\eta^{(n)}$. Tangential forces $\boldsymbol{f}^{(t)}$ may either stick or slip depending on whether a Coulomb friction criterion, with particle friction constant $\mu_{p}$, is satisfied. Stick interactions are defined as those with $\left|\boldsymbol{f}^{(t)}\right|<\mu_{p}\left|\boldsymbol{f}^{(n)}\right|$ and, like the normal force, are calculated from a linear spring-dashpot with parameters $k^{(t)}$ and $\eta^{(t)}$. Interactions with greater computed tangential forces are labelled as slip events and the tangential force is truncated to $\left|\boldsymbol{f}^{(t)}\right|=\mu_{p}\left|\boldsymbol{f}^{(n)}\right|$. In this paper parameters are chosen which give $k^{(n)} / p>10^{4}$ so that calculations are in the rigid particle regime of da Cruz et al. (2005). Unless stated otherwise the values $\mu_{p}=0.4, k^{(n)}=10^{4}$ and $k^{(t)}=0.5 k^{(n)}$ are used. Particle interactions with these values are very short-lived so that results are invariant of the viscous dissipation. The tangential dashpot is therefore neglected $\left(\eta^{(t)}=0\right)$ and $\eta^{(n)}=4.2$ is chosen, as in Silbert et al. (2001), so that the particles have a restitution coefficient of $e=0.9$.

To select a mean packing fraction $\phi_{0}$, the system size along the $\hat{x}$ direction is determined as $L=\bar{A} N /\left(H \phi_{0}\right)$, where $\bar{A}$ is the average grain area. The shear cell is then populated with $N$ particles with density $\rho_{*}$ and mean diameter $d$ and $V_{0}$ set to unity in order to match the non-dimensional iCIDR equations. In order to avoid crystallization effects, the individual particle diameters are chosen randomly from a discretized distribution. Here an even spread from $0.8 d, 0.9 d, d, 1.1 d$ and $1.2 d$ is taken so that the number of particles of each diameter is $N / 5$. These particles, which are initially elastic but not frictional, are randomly distributed in the domain. This results in overlaps which would normally cause very large elastic forces, so firstly there is a period during which the total kinetic energy of all particles is scaled to a small constant value so that the system can reach an equilibrium state. The arrangement which results from this procedure has almost uniform packing density and very small overlaps. Then, the interaction algorithm is altered so that the particles are approximately rigid (very large $k^{(n)}$ ) and frictional. The true simulation begins after the velocity fields are prescribed.

The steady-state $\mu(I)$ and $\Phi(I)$ relations found in previous works (e.g. da Cruz et al. 2005) are first confirmed in order to obtain macroscopic rheological parameters. Both curves are derived from the same set of experiments in which the solids volume fractions $\phi_{0}$ take values in the range 0.76-0.8. This range is expected to lie in the inertial regime and, due to the $\Phi(I)$ relation, each packing corresponds to a unique inertial number. Once the system has reached a steady state, the flow fields are coarse-grained. This is achieved by averaging in the $\hat{x}$ direction and then averaging 
within bins which discretize $\hat{z}$ into boxes of height $2 d$. Each run is then repeated 10 times in order to calculate error estimates. The solids volume fraction $\phi$ and the two velocity components $\hat{u}$ and $\hat{w}$ are clearly defined in the DEM data and allow the inertial number to be readily calculated from its definition (1.1). Calculation of the bulk friction coefficient $\mu$ requires the macroscopic stress components to be defined. As in Silbert et al. (2001) this involves a sum over all particles $\alpha$ in the sampling volume:

$$
\sigma_{i j}=\frac{1}{2 d L} \sum_{\alpha}\left[\sum_{\beta>\alpha} r_{i}^{\alpha \beta} f_{j}^{\alpha \beta}+m^{\alpha} \delta \hat{u}_{i}^{\alpha} \delta \hat{u}_{j}^{\alpha}\right],
$$

where $\boldsymbol{r}^{\alpha \beta}$ is the centre-to-centre vector, $\boldsymbol{f}^{\alpha \beta}$ is the total force between particle pairs and $\delta \hat{\boldsymbol{u}}^{\alpha}=\left(\hat{u}^{\alpha}-\hat{V}_{0} \hat{z} / \hat{H}, \hat{w}^{\alpha}\right)$ is the velocity fluctuation of particle $\alpha$. From the stress decomposition (2.3) these stress components are used to calculate the pressure $p$, deviatoric stress $\|\boldsymbol{\tau}\|$ and hence $\mu$ across the domain. As these quantities are all found to be invariant of $\hat{z}$, the mean value is taken for the $\mu(I)$ data presented in figure 1(a). The volume fraction is also found to be constant at steady state so that the $\Phi(I)$ relation, plotted in figure $1(b)$, is simply verified using the mean packing density $\Phi=\phi_{0}$.

\section{REFERENCES}

BAgNOLD, R. A. 1954 Experiments on a gravity-free dispersion of large solid spheres in a Newtonian fluid under shear. Proc. R. Soc. Lond. A 225 (1160), 49-63.

BARKER, T. \& GRAY, J. 2017 Partial regularisation of the incompressible $\mu(I)$-rheology for granular flow. J. Fluid Mech. 828, 5-32.

Barker, T., Schaeffer, D. G., Bohorquez, P. \& Gray, J. M. N. T. 2015 Well-posed and ill-posed behaviour of the $\mu(I)$-rheology for granular flow. J. Fluid Mech. 779, 794-818.

Barker, T., Schaeffer, D. G., Shearer, M. \& Gray, J. M. N. T. 2017 Well-posed continuum equations for granular flow with compressibility and $\mu(I)$-rheology. Proc. R. Soc. Lond. A 473 (2201), 20160846.

Bouzid, M., Trulsson, M., Claudin, P., Clément, E. \& Andreotti, B. 2013 Nonlocal rheology of granular flows across yield conditions. Phys. Rev. Lett. 111 (23), 238301.

Canuto, C., Hussaini, M. Y., Quarteroni, A. \& Zang, T. A. 1988 Spectral Methods in Fluid Dynamics, Springer Series in Computational Physics. Springer.

Chialvo, S., Sun, J. \& Sundaresan, S. 2012 Bridging the rheology of granular flows in three regimes. Phys. Rev. E 85 (2), 021305.

Da Cruz, F., Emam, S., Prochnow, M., Roux, J. \& Chevoir, F. 2005 Rheophysics of dense granular materials: discrete simulation of plane shear flows. Phys. Rev. E 72, 021309.

Cundall, P. A. \& StRACK, O. D. 1979 A discrete numerical model for granular assemblies. Geotechnique 29 (1), 47-65.

DAerr, A. \& Douady, S. 1999 Two types of avalanche behaviour in granular media. Nature 399, 241-243.

DeGiuli, E. \& Wyart, M. 2017 Friction law and hysteresis in granular materials. Proc. Natl Acad. Sci. USA 114, 9284-9289.

Edelen, D. G. 1972 A nonlinear Onsager theory of irreversibility. Intl J. Engng Sci. 10 (6), 481-490.

EdWARDS, A. N. \& GRAY, J. M. N. T. 2015 Erosion-deposition waves in shallow granular freesurface flows. J. Fluid Mech. 762, 35-67.

Edwards, A. N., Viroulet, S., Kokelaar, B. P. \& Gray, J. M. N. T. 2017 Formation of levees, troughs and elevated channels by avalanches on erodible slopes. J. Fluid Mech. 823, 278-315.

Forterre, Y. \& Pouliquen, O. 2008 Flows of dense granular media. Annu. Rev. Fluid Mech. 40, 1-24. 
GDR MiDi 2004 On dense granular flows. Eur. Phys. J. E 14 (4), 341-365.

Goddard, J. D. \& LEE, J. 2018 Regularization by compressibility of the $\mu(I)$ model of dense granular flow. Phys. Fluids 30, 073302.

GraY, J. M. N. T. \& EDWARDS, A. N. 2014 A depth-averaged $\mu(I)$-rheology for shallow granular free-surface flows. J. Fluid Mech. 755, 503-534.

Heyman, J., Delannay, R., Tabuteau, H. \& Valance, A. 2017 Compressibility regularizes the $\mu(I)$-rheology for dense granular flows. J. Fluid Mech. 830, 553-568.

JACKSON, R. 1983 Some mathematical and physical aspects of continuum models for the motion of the granular materials. In Theory of Dispersed Multiphase Flow (ed. R. Meyer). Academic Press.

Jop, P., Forterre, Y. \& Pouliquen, O. 2006 A constitutive relation for dense granular flows. Nature 44, 727-730.

Kamrin, K. \& Koval, G. 2012 Nonlocal constitutive relation for steady granular flow. Phys. Rev. Lett. 108 (17), 178301.

Lagrée, P.-Y., Staron, L. \& Popinet, S. 2011 The granular column collapse as a continuum: validity of a two-dimensional Navier-Stokes model with a $\mu(I)$-rheology. J. Fluid Mech. 686, 378-408.

LEes, A. \& EDWARDS, S. 1972 The computer study of transport processes under extreme conditions. J. Phys. C: Solid State Phys. 5 (15), 1921-1928.

LiU, A. \& Nagel, S. 1998 Jamming is not just cool any more. Nature 396, 21-22.

Martin, N., Ionescu, I. R., Mangeney, A., Bouchut, F. \& Farin, M. 2017 Continuum viscoplastic simulation of a granular column collapse on large slopes: $\mu(I)$ rheology and lateral wall effects. Phys. Fluids 29, 013301.

OTsuki, M. \& HaYAKawa, H. 2009 Critical behaviors of sheared frictionless granular materials near the jamming transition. Phys. Rev. E 80 (1), 011308.

OTSUKi, M. \& HAYAKAWA, H. 2011 Critical scaling near jamming transition for frictional granular particles. Phys. Rev. E 83 (5), 051301.

Pouliquen, O., Cassar, C., Jop, P., Forterre, Y. \& Nicolas, N. 2006 Flow of dense granular material: towards simple constitutive laws. J. Stat. Mech. 2006, P07020.

Pouliquen, O. \& Forterre, Y. 2002 Friction law for dense granular flows: application to the motion of a mass down a rough inclined plane. J. Fluid Mech. 453, 133-151.

Pouliquen, O.\& Forterre, Y. 2009 A non-local rheology for dense granular flows. Phil. Trans. R. Soc. Lond. A 367, 5091-5107.

Russell, A. S., Johnson, C. G., Edwards, A. N., Viroulet, S., Rocha, F. M. \& Gray, J. M. N. T. 2019 Retrogressive failure of a static granular layer on an inclined plane. J. Fluid Mech. 869, 313-340.

SCHIESSER, W. E. 2012 The Numerical Method of Lines: Integration of Partial Differential Equations. Elsevier.

Schofield, A. \& Wroth, P. 1968 Critical State Soil Mechanics, vol. 310. McGraw-Hill.

Silbert, L. E., Ertaş, D., Grest, G. S., Halsey, T. C., Levine, D. \& Plimpton, S. J. 2001 Granular flow down an inclined plane: Bagnold scaling and rheology. Phys. Rev. E 64 (5), 051302 .

Singh, A., Magnanimo, V., Saitoh, K. \& Luding, S. 2015 The role of gravity or pressure and contact stiffness in granular rheology. New J. Phys. 17 (4), 043028.

Staron, L., Lagrée, P.-Y. \& Popinet, S. 2012 The granular silo as a continuum plastic flow: the hour-glass versus the clepsydra. Phys. Fluids 24, 103301.

Trefethen, L. N. 2000 Spectral Methods in MATLAB, Software, Environments, and Tools, vol. 10. Society for Industrial and Applied Mathematics (SIAM).

Trulsson, M., Bouzid, M., Claudin, P. \& Andreotti, B. 2013 Dynamic compressibility of dense granular shear flows. Eur. Phys. Lett. 103, 38002. 